\title{
Nonlinear coupling of photons via a collective mode of transparent superconductor
}

\author{
V. M. Akulin ${ }^{1,2,3}$ \\ ${ }^{1}$ Laboratoire Aimé Cotton, CNRS (UPR 3321), Bâtiment 505, 91405 Orsay Cedex, France. \\ ${ }^{2}$ Institute for Information Transmission Problems of the Russian Academy of Science, \\ Bolshoy Karetny per. 19, Moscow, 127994, Russia. \\ ${ }^{3}$ Laboratoire J.-V. Poncelet CNRS (UMI 2615) Bolshoi Vlassievsky per. 11, Moscow, 119002 Russia.
}

\begin{abstract}
At the first glance, the expression "transparent superconductor" may seem an oxymoron. Still, the first principle calculations [1] and experiments 2] show that the materials that behave as superconductors at low frequencies and do not absorb in the optical domain may exist. Virtual excitation of the collective electronic modes of such superconductors in a magnetic field appears as an efficient way to realize the nonlinear interaction of light at the level of two single photons. The essence of the effect is in the fact that the pondermotor energy is proportional to the ratio of the charge squared to the mass of the "collective particle" interacting with radiation, $e^{2} / m$, and therefore, for a "particle" representing a collective motion of many electrons, it scales linearly-, and its secondorder correction quadratically with the number of the electrons involved. This general situation is analyzed in detail in the framework of a simple model of a fiber tube waveguide equipped with a clean superconductor layer. It turns out that for realistic parameters, at the $\mu$-scale of the tube diameter and the $\mathrm{cm}$-scale of the fiber length, such a system is capable of performing the logic gate operation on the polarization variables of a pair of optical photons.
\end{abstract}

PACS numbers: 03.65.-w Quantum mechanics, 42.65.Wi Nonlinear waveguides, 78.20.Bh Theory, models, and numerical simulation.

Interaction of photons mediated by atomic or condensed-matter electrons gets stronger when the latter are in a collective or a cooperative [3] quantum state. Excitations of the Cooper pair condensate in superconductors, whose interaction with photons is well-described since long ago [4, [5], [6], is one of the examples of such collective states. It will be shown here, that these states in "transparent superconductors" can mediate a rather strong coupling of a pair of single photons. It might happen that the optically transparent and superconducting substance required for the purpose does not yet exist, but can be predicted by the first principle calculations, like it has been done 1] for one of the candidates $-p$-doped $\mathrm{CuAlO}_{2}$. Searching for such materials is worth to be done in view of the importance of the visible or near infrared light manipulation at the level of single photons for Quantum Informatics. The present paper starts with consideration of interaction of photons with transparent materials followed by calculation of the nonlinear susceptibility of a "clean" superconductor and detailed for a specific setting of photon propagation in a tube waveguide enveloping a thin superconducting layer.

The non-relativistic Pauli equation for an electron in an external quantized electromagnetic field suggests the interaction term in the form $-\frac{e}{m c} \widehat{\vec{p}} \widehat{\vec{A}}+\frac{e^{2} \widehat{\vec{A}}^{2}}{2 m c^{2}}$, where the vector potential operator

$$
\widehat{\vec{A}}(\vec{r})=\sum_{k} \sqrt{\frac{c \pi \hbar v}{\omega_{k}}}\left(\widehat{a}_{k} \vec{u}_{k}(\vec{r})+\widehat{a}_{k}^{+} \vec{u}_{k}^{*}(\vec{r})\right)
$$

is given in terms of photon frequency $\omega_{k}$, the group velocity $v[7]$, and the creation $\widehat{a}_{k}^{+}$and annihilation $\widehat{a}_{k}$ operators of the photons with the mode functions $\vec{u}_{k}(\vec{r})$ normalized by the volume integral $\int \vec{u}_{k} \vec{u}_{k}^{*} d V=1$. The scalar product is implicit.
From the viewpoint of the relativistic Dirac equation, the pondermotor term $e^{2} \widehat{\vec{A}}^{2} / 2 m c^{2}$ containing the square of the electron-photon interaction divided by the energy of an electron-positron pair at rest can be interpreted as a second order relativistic perturbation, usually small, unless the electromagnetic field is really strong, as for the case of multiphoton laser ionization of atoms [8]. However, for transparent materials, where no resonant levels are available for optical transitions, the main term $-\frac{e}{m c} \widehat{\vec{p}} \widehat{\vec{A}}$ also gives just a second order contribution, which is yet smaller than $e^{2} \widehat{\vec{A}}^{2} / 2 m c^{2}$ by a factor $\sim v_{F} / c$ - the ratio of the Fermi and the light velocities. See [ Appendix for the details of the estimate.

For a multi-electron system with the electron density $n_{e}$, the interaction can be written as $n_{e} e^{2} \vec{A}^{2} / 2 m c^{2} \equiv$ $\left(\omega_{p} / \omega_{k}\right)^{2} \vec{E}^{2} / 8 \pi$, where $\omega_{p}=\sqrt{4 \pi n_{e} e^{2} / m}$ is the plasma frequency. Even for not absorbing media, the electromagnetic field at frequencies $\omega_{k}<\omega_{p}$ can penetrate at most at the length $\lambda_{p} \sim 2 \pi \omega_{k} / c \sqrt{\left(\omega_{p} / \omega_{k}\right)^{2}-1}$, which implies that at least one of the spacial dimensions of the superconductor should be less than $\lambda_{p}$ for the transparence required. For a pair of photons at close frequencies $\omega_{k}$ and $\omega_{k^{\prime}}$ far detuned from two-photon resonances, in the pondermotor interaction term

$$
\widehat{n}_{e} \frac{e^{2} \pi \hbar v}{2 m c} \sum_{k ; k^{\prime}} \frac{\widehat{a}_{k} \widehat{a}_{k^{\prime}}^{+} \vec{u}_{k^{\prime}}^{*}(\vec{r}) \vec{u}_{k}(\vec{r})+h . c .}{\sqrt{\omega_{k^{\prime}} \omega_{k}}},
$$

one can retain only the terms oscillating at the photon frequency difference, which can be tuned close to the resonance with collective modes of the superconductor. Here $\widehat{n}_{e}=\widehat{\psi}^{\dagger}(\vec{r}) \widehat{\psi}(\vec{r})$ is the electron density operator given 
in terms of the anticommuting electron creation $\widehat{\psi}^{\dagger}(\vec{r})$ and annihilation $\widehat{\psi}(\vec{r})$ field operators, and h.c. denotes Hermite conjugate.

Consider now such a system for the case of a superconductor at zero temperature in a static sub-critical magnetic field given by the vector potential $\vec{A}_{s t}$. Each of the photons is in a superposition of longitudinal modes at close frequencies $\omega_{k} \cong \omega$ and $\omega_{k^{\prime}} \cong \omega+\delta \omega$, respectively, such that $\hbar \delta \omega$ is less than the gap parameter $\Delta(\vec{r})$. The atomic units $m=1, \hbar=1, e=1$ are employed hereafter for shortness. In the framework of the model with a local coupling $-g$, the corresponding Hamiltonian reads

$$
\begin{aligned}
\widehat{H} & =\int d V\left[\frac{1}{2} \widehat{\psi}_{s}^{\dagger}(\vec{r})\left(\widehat{\vec{p}}-\widehat{\vec{A}}_{s t} / c\right)^{2} \widehat{\psi}_{s}(\vec{r})\right. \\
& -\frac{1}{2} \widehat{\Delta}(\vec{r}) \widehat{\psi}_{s}^{\dagger}(\vec{r}) \widehat{\psi}_{-s}^{\dagger}(\vec{r})-\frac{1}{2} \widehat{\Delta}^{\dagger}(\vec{r}) \widehat{\psi}_{s}(\vec{r}) \widehat{\psi}_{-s}(\vec{r}) \\
& +\frac{1}{2 g} \widehat{\Delta}^{\dagger}(\vec{r}) \widehat{\Delta}(\vec{r})+\frac{\pi v}{2 c} \widehat{\psi}_{s}^{\dagger}(\vec{r}) \widehat{\psi}_{s}(\vec{r}) \times \sum_{k ; k^{\prime}} \\
& \left.\frac{\widehat{a}_{k} \widehat{a}_{k^{\prime}}^{+} \vec{u}_{k^{\prime}}^{*}(\vec{r}) \vec{u}_{k}(\vec{r})+h . c .}{\sqrt{\omega_{k^{\prime}} \omega_{k}}}\right]+\sum_{k} \omega_{k}\left(\widehat{a}_{k}^{+} \widehat{a}_{k}+\frac{1}{2}\right)
\end{aligned}
$$

where summation over the spin subscripts $s= \pm$, which denotes $\pm 1 / 2$, is implicit. Magnetic interaction with spins is ignored.

In the case where photons are out of resonance with the superconductor excitations, the interaction among them can be considered as the second order perturbation, such that the photon part of the Hamiltonian adopts the form

$\widehat{H}=\sum_{k} \omega_{k}\left(\widehat{a}_{k}^{+} \widehat{a}_{k}+\frac{1}{2}\right)+\sum_{k k^{\prime}} \chi_{k, k^{\prime}, k^{\prime}, k}(\delta \omega) \widehat{a}_{k} \widehat{a}_{k^{\prime}}^{+} \widehat{a}_{k^{\prime}} \widehat{a}_{k}^{+}$,

which implies that the nonlinear coupling of photons occur via the linear susceptibility of the multielectronic system to the pondermotor perturbation $\widehat{\vec{A}}^{2} / 2 c^{2}$. This nonlinear susceptibility $\chi_{k, k^{\prime}, k^{\prime}, k}$ of the transparent superconductor is the very quantity to be calculated.

It is convenient to invoke a standard technique - the Feynmann integration over the anticommuting electron fields and classical fields for the order parameter [9]. In the framework of this approach,

$$
\chi_{k, k^{\prime}, k^{\prime}, k}=\frac{\partial^{2}}{\partial \alpha \partial \alpha^{*}} \ln Z\left(\alpha^{*}, \alpha\right),
$$

where the "partition function" $Z$ is given by the functional integral in the momentum representation

$$
Z=\int e^{i S} D \psi_{+}^{*} D \psi_{+} D \psi_{-}^{*} D \psi_{-} D \Delta_{1}^{*} D \Delta_{1} D \Delta_{2}^{*} D \Delta_{2}
$$

with the action

$$
\begin{aligned}
S & =\int\left(\begin{array}{llll}
\psi_{+}^{*} & \psi_{+}^{*} & \psi_{-} & \psi_{-}
\end{array}\right) \widehat{M}\left(\begin{array}{c}
\psi_{+} \\
\psi_{+} \\
\psi_{-}^{*} \\
\psi_{-}^{*}
\end{array}\right) \frac{d \widetilde{\omega}}{2} d^{3} \widetilde{k} \\
& +\int \frac{1}{2 g} \delta \Delta^{*}(\omega, \vec{k}) \delta \Delta(\omega, \overrightarrow{\vec{k}}) d \widetilde{\omega} d^{3} \widetilde{k}
\end{aligned}
$$

The Hamiltonian Eq.(3) corresponds to the matrix $\widehat{M}$ of the form [10]

$$
\left(\begin{array}{cccc}
\delta \omega+\widetilde{\omega}-\epsilon_{1} & \alpha^{*} \vec{u}_{k}^{*} \vec{u}_{k^{\prime}} & -\Delta_{1} & -\Delta \\
\alpha \vec{u}_{k^{\prime}}^{*} \vec{u}_{k} & \widetilde{\omega}-\epsilon_{2} & -\Delta & -\Delta_{2} \\
-\Delta^{*}{ }_{1} & -\Delta^{*} & \widetilde{\omega}+\epsilon_{3} & -\alpha \vec{u}_{k^{\prime}}^{*} \vec{u}_{k} \\
-\Delta^{*} & -\Delta_{2}^{*} & -\alpha^{*} \vec{u}_{k}^{*} \vec{u}_{k^{\prime}} & \delta \omega+\widetilde{\omega}^{2}+\epsilon_{4}
\end{array}\right)
$$

Here $\alpha$ and $\alpha^{*}$ replace the operators $\widehat{a}_{k} \widehat{a}_{k^{\prime}}^{+}$and $\widehat{a}_{k^{\prime}} \widehat{a}_{k}^{+}$, respectively, $\epsilon_{2}, \epsilon_{3}$ and $\epsilon_{1}, \epsilon_{4}$ are energies of electrons comprising Cooper pairs before and after the photon-induced virtual transition, respectively. The factors $\sqrt{\frac{\pi v}{2 c \omega_{k}}}$ are included to the vectors $\vec{u}_{k}$ for shortness. The order parameter amplitudes $\Delta_{1}, \Delta_{2}$, and $\Delta$ are specified below. See II Appendix for the details of the transformations performed.

It is expedient to discuss the gap parameters of Eq. (8) in some more detail. For superconducting systems at zero temperature not interacting with radiation, the minimum energy attains at a stationary non-zero value of the gap $\Delta(\vec{r})$, with a phase dependent on coordinates in the presence of a non-zero magnetic field switched on before cooling the conductor. For a small perturbation by virtual absorption of circularly polarized photons, this variable also may experience a variation $\delta \Delta=$ $\Delta_{1}(\vec{r})+\Delta_{2}(\vec{r})$, which, in a sense, resembles that of the two-band Leggett model[11], although it couples the pair's electrons with distinct angular momenta but not in different conduction bands. This corresponds to a virtual excitation of non-dissipative collective motion of Cooper pairs in magnetic field at a frequency below $2|\Delta|$, which has resonant structure and chirality due to the absorbed angular momentum, and thereby drastically affect tensor $\chi_{k, k^{\prime}, k^{\prime}, k}$ close to the resonance. For the virtual transition with no change of the angular momentum, the perturbation $\Delta_{2}$ turns to coincide (up to a phase of $\Delta$ ) with $\Delta_{1}{ }^{*}$ and cancels the contribution of the latter, such that no collective virtual excitation occurs.

Consider this situation for a specific setting shown in Fig.1. The superconductor is placed as a cylindric layer of radius $R$ and thickness $d \ll \lambda_{p}$ within a thin wall of a transparent dielectric tube waveguide with the refraction index $n$. The problem thus has to be formulated in cylindrical coordinates $r, \theta, z$ and in the momentum representation $\theta \rightarrow L, z \rightarrow k$. The photon mode functions $\vec{u}_{k}(\vec{r})$ and $\vec{u}_{k^{\prime}}(\vec{r})$ in the the axial symmetry setting 


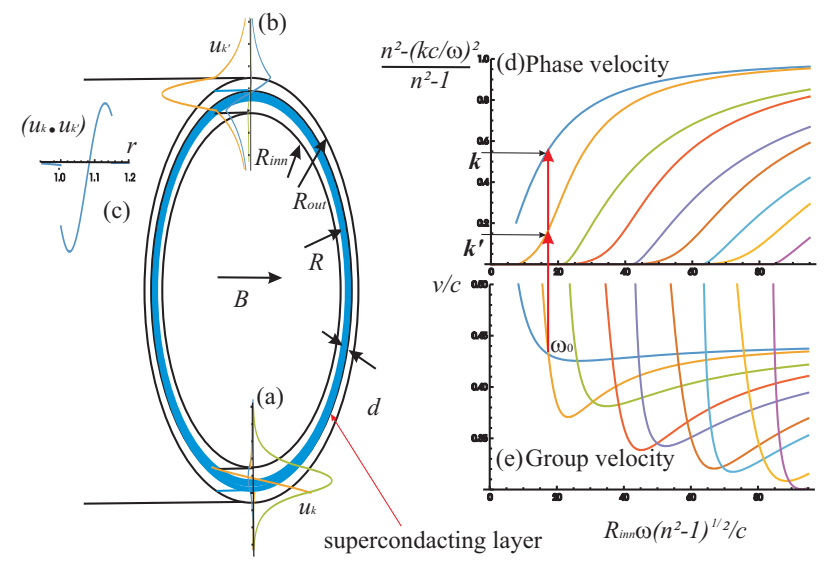

FIG. 1: Degenerate tube modes $L= \pm 1$ for the refraction index $n=2.26$ and the ratio of the outer and inner diameters of the tube $R_{\text {out }} / R_{\text {inn }}=1.15$. In the inlets (a) The mode function of the first mode. The strong symmetric component $u_{\theta}$, the asymmetric $-u_{r}$. (b) The second mode function. The strongest symmetric component $u_{r}$, the asymmetric component $u_{z}$. (c) The scalar product of the mode functions. (d) and (e) The phase and the group velocities, respectively. On the abscise axis - scaled frequency $R_{i n n} \frac{\omega}{c} \sqrt{n^{2}-1}$, on the ordinate axis of $(\mathrm{d})$ - scaled and shifted phase velocity $\frac{\frac{k c}{\omega}-1}{n-1}$. The "working point" is near the frequency $\omega_{0}$ where the group velocities coincide, while the phase velocities and the corresponding wavevectors $k$ and $k^{\prime}$ are different.

have the coordinate dependencies

$$
\begin{aligned}
\sqrt{\frac{\pi v}{2 l c \omega_{k}}} \vec{u}_{k}(r, z, \theta) & =\left(\begin{array}{c}
u_{z}(r) \\
u_{r}(r) \\
u_{\theta}(r)
\end{array}\right) e^{-i \omega t+i k z+i L \theta} \\
\sqrt{\frac{\pi v}{2 l c \omega_{k^{\prime}}}} & \vec{u}_{k^{\prime}}(r, z, \theta)=\left(\begin{array}{c}
u_{z}^{\prime}(r) \\
u_{r}^{\prime}(r) \\
u_{\theta}^{\prime}(r)
\end{array}\right) e^{-i \omega^{\prime} t+i k^{\prime} z+i L^{\prime} \theta}
\end{aligned}
$$

that correspond to two modes chosen to have close group velocities $v$ and $v^{\prime}$. Here, the aforesaid factor $\sqrt{\frac{\pi v}{2 c \omega_{k}}}$ also includes the normalization to a waveguide length $l$. The radial mode functions $u_{z}(r)=-b q Z_{L}(r q) / 2 k, u_{\theta}(r)=$ $b L Z_{L}(r q) / r q-a Z_{L}^{\prime}(r q)$, and $u_{r}(r)=a i L Z_{L}(r q) / r q-$ bi $Z_{L}^{\prime}(r q)$ are given in terms of the Bessel functions: $Z_{L}=$ $K_{L}$ outside -, $Z_{L}=I_{L}$ inside - , and $Z_{L}=\gamma J_{L}+\varkappa Y_{L}$ within the tube wall, and are normalized by the condition $\int 2 \pi r|u|^{2} d r=1$. The parameter $q$ amounts to $\sqrt{k^{2}-\omega_{k}^{2} c^{-2}}$ and $\sqrt{n^{2} \omega_{k}^{2} c^{-2}-k^{2}}$ outside and inside the wall, respectively. The dispersion curves $\omega_{k}(k)$ and $\omega_{k^{\prime}}\left(k^{\prime}\right)$ correspond to different waveguide modes that are found numerically from the boundary conditions at the inner and outer radii of the tube wall. The thin transparent superconducting layer is ignored in the consideration of the mode fields. See III Appendix for the details of the calculations.
For the electron energies, the cylindrical symmetry implies

$$
\epsilon_{f}(\widetilde{L}, \widetilde{k})=\frac{p_{r}^{2}}{2}+\frac{\widetilde{k}^{2}}{2}+\frac{(\widetilde{L}-\bar{L})^{2}}{R^{2}}-\mu,
$$

where $\widetilde{k}$ is the momentum along the axis, $\widetilde{L}$ is the angular momentum, $p_{r}$ is the radial momentum, $\mu$ is the chemical potential chosen as the reference energy. The stationary azimuthal magnetic field potential $\vec{A}_{s t}$ allowing for the magnetic field parallel to the cylinder axis is parametrized by the number $\bar{L}$ of the magnetic field quanta traversing the tube cross-section. Note that $\bar{L}$ accounts here for the magnetic field potential not compensated by the persistent currents.

If a magnetic field corresponding to $\bar{L}=\Lambda$ was passing through the waveguide tube cross-section before the layer was cooled down to the superconducting state, than after the cooling, the magnetic field remains the same while the stationary order parameter $\Delta$ gets the angular dependence $e^{i 2 \Lambda \theta}$ corresponding to zero persistent currents. If after the cooling, the magnetic field has been further augmented, up to the value characterized by the parameter $\bar{L}$, the order parameter angular dependence remains the same, but there appears a persistent current compensating the augmentation of the magnetic flux through the cross-section, such that the angular momenta of the electrons now acquire a shift by the final value of $\bar{L}$. For a thin superconducting layer one can ignore the radial dependence of $\Delta(\vec{r})$.

Now one can explicitly find the energies

$$
\begin{aligned}
& \epsilon_{1}=\epsilon_{f}(\widetilde{L}+\delta L, \widetilde{k}+\delta k) \\
& \epsilon_{2}=\epsilon_{f}(\widetilde{L}, \widetilde{k}) \\
& \epsilon_{3}=\epsilon_{f}(2 \Lambda-\widetilde{L},-\widetilde{k}) \\
& \epsilon_{4}=\epsilon_{f}(2 \Lambda-\widetilde{L}-\delta L,-\widetilde{k}-\delta k),
\end{aligned}
$$

entering Eq.(8) and the small perturbation

$\delta \Delta=\frac{\Delta_{1} e^{i z \delta k-i t \delta \omega+i \theta(\delta L+2 \Lambda)}+\Delta_{2} e^{-i z \delta k+i t \delta \omega-i \theta(\delta L-2 \Lambda)}}{\sqrt{2 \pi d R l}}$

normalized to the layer volume. Here $\delta k=k-k^{\prime}$ and $\delta L=L-L^{\prime}$ depend on the photon modes and $\Delta_{1,2}$ are the amplitudes entering Eq.(8).

Further a bit cumbersome but completely straightforward calculations can be sketched as follows. Integration over the anticommuting fields $\psi$ yields

$$
Z=\int e^{\int \frac{d \tilde{\omega}}{2} \operatorname{Tr}[\log (\operatorname{det} \widehat{M})]+i \frac{\Delta_{1}^{*} \Delta_{1}+\Delta_{2}^{*} \Delta_{2}}{2 g}} d \Delta_{1} \ldots d \Delta_{2}^{*},
$$

where the first term in the exponent at the right hand side serves as an action for the variables $\Delta_{1}$ and $\Delta_{2}$ with $\widehat{M}$ given by Eqs. (811). After being cast in Taylor series up to the second order, integrated over the frequency $d \widetilde{\omega}$ 
and traced, this term reads

$$
\int d \widetilde{\omega} \operatorname{Tr}[\log (\operatorname{det} \widehat{M})] \simeq\left(\begin{array}{lll}
\alpha & \Delta_{1} & \Delta_{2}^{*}
\end{array}\right) \widehat{\widetilde{\mathcal{M}}}\left(\begin{array}{c}
\alpha^{*} \\
\Delta_{1}^{*} \\
\Delta_{2}
\end{array}\right)
$$

with

$$
\widehat{\widetilde{\mathcal{M}}}=\left(\begin{array}{ccc}
\widetilde{\mathcal{M}}_{\alpha, \alpha} & -\widetilde{\mathcal{M}}_{\Delta, \alpha} & \widetilde{\mathcal{M}}_{\Delta, \alpha} \\
-\widetilde{\mathcal{M}}_{\Delta, \alpha} & \widetilde{\mathcal{M}}_{\Delta, \Delta} & \widetilde{\mathcal{M}}_{\Delta, \bar{\Delta}} \\
\widetilde{\mathcal{M}}_{\Delta, \alpha} & \widetilde{\mathcal{M}}_{\Delta, \bar{\Delta}} & \widetilde{\mathcal{M}}_{\Delta, \Delta}
\end{array}\right),
$$

where the matrix elements

$$
\begin{gathered}
\widetilde{\mathcal{M}}_{\alpha, \alpha}=-2 i \nu O_{p} \mathcal{I}_{1} \\
\widetilde{\mathcal{M}}_{\Delta, \Delta}=i \nu O_{o}\left(\mathcal{I}_{2}+\mathcal{I}_{4}\right) \\
\widetilde{\mathcal{M}}_{\Delta, \alpha}=-i \nu O_{p o} \mathcal{I}_{3} \\
\widetilde{\mathcal{M}}_{\Delta, \bar{\Delta}}=i \nu O_{o} \mathcal{I}_{4}
\end{gathered}
$$

are given in terms of the integrals

$$
\begin{aligned}
& \mathcal{I}_{1}(\Omega)=\left(\int_{D[J]} \frac{(\cosh (\xi-\varsigma)+1) d \xi d \varsigma}{\Omega+\cosh \xi+\cosh \varsigma}\right)_{+} \\
& \mathcal{I}_{2}(\Omega)=\left(\int_{D[J]} \frac{\left(-e^{\xi+\varsigma}-1\right) d \xi d \varsigma}{\Omega+\cosh \xi+\cosh \varsigma}\right)_{+} \\
& \mathcal{I}_{3}(\Omega)=\left(\int_{D[J]} \frac{\left(e^{\xi}+e^{\varsigma}\right) d \xi d \varsigma}{\Omega+\cosh \xi+\cosh \varsigma}\right)_{-} \\
& \mathcal{I}_{4}(\Omega)=\left(\int_{D[J]} \frac{d \xi d \varsigma}{\Omega+\cosh \xi+\cosh \varsigma}\right)_{+},
\end{aligned}
$$

and where the subscripts \pm denote sum or difference of the integrals in the parentheses for the positive and the negative scaled frequency $\Omega= \pm \frac{\delta L(\Lambda-\bar{L}) / R^{2}-\Delta \omega}{|\Delta|}$, respectively. The functions $\mathcal{I}_{j}(\Omega)$ diverge logarithmically at the gap edges $|\Omega|=2$.

The other quantities entering Eq.(16) are the mode overlap functions

$$
\begin{aligned}
O_{p} & =\frac{(\pi v / c)^{2}}{2 \omega_{k^{\prime}} \omega_{k}} \frac{\pi R d\left(\vec{u}_{k}^{*}(R) \cdot \vec{u}_{k^{\prime}}(R)\right)^{2}}{l} \\
O_{p o} & =\frac{\pi v / c}{\sqrt{2 \omega_{k} \omega_{k^{\prime}}}} \frac{\sqrt{\pi R d}\left(\vec{u}_{k}^{*}(R) \cdot \vec{u}_{k^{\prime}}(R)\right)}{\sqrt{l}} \\
O_{o} & =1
\end{aligned}
$$

with the restored pre-factors. Since the integrals Eq.(17) originate from the tracing in Eq.(14) replaced by the integration over the phase volume $\operatorname{Tr}[\ldots] \rightarrow$ $\sum_{\widetilde{L}} \int \ldots \frac{n_{e} d V d p_{r} d \widetilde{k}}{R(2 \pi)^{3}}$, the factor

$$
\nu=\frac{|\Delta| n_{e}}{8 \pi \sqrt{\frac{\delta L^{2}}{R^{2}}+\delta k^{2}}}
$$

in Eq.(16) is the Jacobian $J$ corresponding to the change of the phase space integration variables

$$
p_{r}, \widetilde{k} \rightarrow\left\{\begin{array}{c}
\xi=\operatorname{arcsinh} \frac{\epsilon_{2}\left(p_{r}, \widetilde{k}\right)+\epsilon_{3}\left(p_{r}, \widetilde{k}\right)}{2|\Delta|} \\
\varsigma=\operatorname{arcsinh} \frac{\epsilon_{1\left(p_{r}, \tilde{k}\right)+\epsilon_{4\left(p_{r}, \widetilde{k}\right)}}^{2|\Delta|}}{}
\end{array}\right.
$$

summed over the angular momentum and divided by $\cosh \zeta \cosh \xi$ and by $2|\Delta|$ as the result of introducing dimensionless frequency, while the integration $d \xi d \varsigma$ is restricted to the domain $D[J]$ where $J$ is real.

The domain $D[J]$ can be explicitly expressed in terms of the variables $\xi$, $\varsigma$, and two parameters $\widetilde{\mu}=\frac{\mu}{|\Delta|}-\frac{(\bar{L}-\Lambda)^{2}}{2 R^{2}|\Delta|}$ and $\kappa=\frac{|\Delta|}{4\left(\delta k^{2}+\delta L^{2} / R^{2}\right)}$. The integration over $\frac{\xi+\varsigma}{2}$ in Eq.(17) can be done analytically yielding a cumbersome but explicit expression dependent on the other integration variable $\xi-\varsigma$ and these parameters, while the integration over $\xi-\varsigma$ has to be done numerically. See IV Appendix for the details of the calculations.

After having performed Gaussian integration Eq. (13) allowing for Eq.(14), from Eq.(15) one obtains the required nonlinear susceptibility

$$
\chi_{k, k^{\prime}, \bar{k}^{\prime}, \bar{k}}=\frac{-|\Delta| n_{e} d\left(\vec{u}_{k}^{*}(R) \cdot \vec{u}_{k^{\prime}}(R)\right)^{2}(v / c)^{2}}{32 l R \omega_{k^{\prime}} \omega_{k} \sqrt{\delta L^{2} / R^{2}+\delta k^{2}}} h(\Omega)
$$

which couples photons with the wave-vectors $k, k^{\prime}, \bar{k}^{\prime}, \bar{k}$ satisfying the condition $k-k^{\prime}=\bar{k}-\bar{k}^{\prime}=\delta k, L-L^{\prime}=$ $\bar{L}-\bar{L}^{\prime}=\delta L$. The frequency profile

$$
h(\Omega)=\mathcal{I}_{1}(\Omega)+\frac{\left(1-\delta_{\delta L}^{0}\right) \mathcal{I}_{3}^{2}(\Omega)}{\mathcal{I}_{2}(\Omega)+\frac{4 \pi \sqrt{\delta k^{2}+\delta L^{2} / R^{2}}}{\Delta g n_{e}}}
$$

is given in terms of the integrals Eq. (17) and the Kronekker delta $\delta_{i}^{j}$, which accounts for the fact that for $\delta L=0$ the collective amplitudes $\Delta_{1,2}$ coincides (up to a phase of $\Delta$ ) with $\Delta_{2,1}^{*}$ and give no net contribution.

For a particular case specified in the figure caption, the calculations Eqs.(1819) result in the profiles shown in Fig 2. One sees a strong resonance of the nonlinear susceptibility around the position of the collective mode, where the denominator in Eq.(19) tends to zero. However, such a situation is only possible in a rather narrow domain of the superconductor parameters where

$$
g n_{e} \Delta \lesssim \frac{4 \pi \sqrt{\delta k^{2}+\delta L^{2} / R^{2}}}{\left|\mathcal{I}_{2}(\Omega=0)\right|},
$$

which depends on the superconducting tube radius and the chosen mods. Due to the logarithmic character of the dependence $\mathcal{I}_{2}(\Omega)$ near $|\Omega|=2$, the position of the resonance becomes exponentially close to the band gap edges when the left hand sides of Eq.(20) is considerably smaller than the right one. For a rough estimation with Eq. (20) one can take $\mathcal{I}_{2}(\Omega=0) \simeq 10$. See $\mathrm{V}$ Appendix for the details of the calculations.

For the case of two different degenerate tube modes each of which carries just a single photon in a linear combination of the left $L=-1$ and the right $L=1$ polarization, the susceptibility is independent of the wave-vectors since $\delta k=k(\omega)-k^{\prime}(\omega)+O\left(\frac{\delta \omega}{c}\right)$ is dominated by the difference of the mode wave-numbers. For a two-photon 


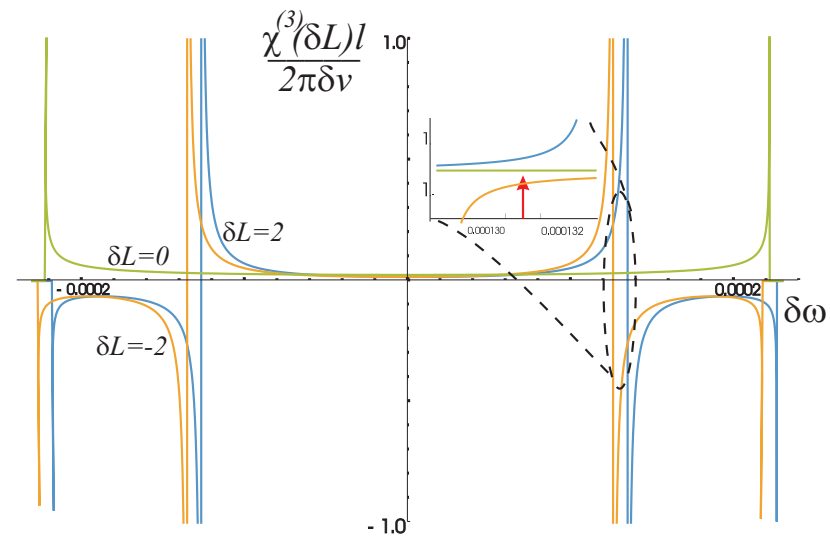

FIG. 2: Collective resonance of $\chi_{\delta L}$ in a transparent superconductor tube of the radius $3 \mu$, the critical temperature $T_{c}=40 \mathrm{~K}$, the electron density $n_{e}=2.57 \times 10^{23} \mathrm{~cm}^{-3}$, the gap $\Delta=2.2 \times 10^{-4}[a . u]$, and the thickness $150 \mathrm{~nm}$, which is a typical London length and the radiation penetration depth $\lambda_{p}$. The chirality of the nonlinear susceptibility is induced by a constant magnetic field of induction $0.01 \mathrm{Ts}$ parallel to the tube axis. The superconductor tube is supported by a tube fiber waveguide with the radii ratio $R_{\text {out }} / R_{\text {inn }}=1.15$, and the refraction index $n=2.26$, for the near infrared light $\lambda=1$ $\mu$. Maximum of the photon vector potentials scalar product $\left|\left(u_{k}^{*}(R) \cdot u_{k^{\prime}}(R)\right)\right|=0.4$ locates at the radius corresponding to the superconductor position $R=1.05 R_{i n n}$. On the basis axis is the detuning frequency $\delta \omega=\omega-\omega^{\prime}$ in atomic units, at the ordinate axis the susceptibilities scaled by the factor $l / 2 \pi \delta v$ with $\delta v=1.33 \times 10^{-2} c$ in order to obtain dimensionless phase shift Eq.(24) in fractions of $\pi$ at the frequency detuning marked by the arrow.

state vector

$$
|\Phi\rangle=\sum_{L, L^{\prime}= \pm 1} \int d z d z^{\prime} \Phi_{L, L^{\prime}}\left(t, z, z^{\prime}\right) \widehat{a}^{\dagger}(z) \widehat{a}^{\prime \dagger}\left(z^{\prime}\right)|0\rangle
$$

given in terms of the operators $\widehat{a}^{\dagger}(z)=\sum_{k} \widehat{a}_{k}^{\dagger} e^{-i k z} / \sqrt{l}$ and $\widehat{a}^{\prime \dagger}(z)=\sum_{k} \widehat{a}_{k^{\prime}}^{\dagger} e^{i k^{\prime} z} / \sqrt{l}$ for the first and the second modes, respectively, the interaction Hamiltonian of Eq.(44) with $\omega_{k} \rightarrow v k, \omega_{k^{\prime}} \rightarrow v^{\prime} k^{\prime}$ results in the Schrödinger equation for the amplitudes $\Phi_{i, j}\left(t, z, z^{\prime}\right)$

$$
\begin{aligned}
& 0=\left(i \widehat{\partial}+\chi_{-2} l \delta_{z-z^{\prime}}\right) \Phi_{-1,-1}\left(t, z, z^{\prime}\right) \\
& 0=\left(i \widehat{\partial}+\chi_{2} l \delta_{z-z^{\prime}}\right) \Phi_{1,1}\left(t, z, z^{\prime}\right) \\
& 0=\left(i \widehat{\partial}+\chi_{0} l \delta_{z-z^{\prime}}\right)\left(\Phi_{1,-1}\left(t, z, z^{\prime}\right)+\Phi_{-1,1}\left(t, z, z^{\prime}\right)\right) \\
& 0=i \widehat{\partial} \Phi_{1,-1}\left(t, z, z^{\prime}\right)-i \widehat{\partial} \Phi_{-1,1}\left(t, z, z^{\prime}\right)=0
\end{aligned}
$$

where $i \widehat{\partial}=-i \frac{\partial}{\partial t}-i v \frac{\partial}{\partial z}-i v^{\prime} \frac{\partial}{\partial z^{\prime}}, \delta_{z-z^{\prime}}$ is the Dirac delta function, and the subscript of $\chi$ denotes $\delta L$ of Eq. (18). See VI Appendix for the details of the calculations.

$$
\begin{aligned}
& \text { The general solution of the equation } \\
& \left(i \widehat{\partial}+A \delta_{z-z^{\prime}}\right) \Phi\left(t, z, z^{\prime}\right)=0 \text { reads } \\
& \Phi\left(t, z, z^{\prime}\right)=\Phi\left(z-v t, z^{\prime}-v^{\prime} t\right) e^{-i \Theta_{z-z^{\prime}} \frac{A}{2 \delta v}},
\end{aligned}
$$

where $\Theta_{z-z^{\prime}}$ is the Haviside step function and $\delta v=$ $v-v^{\prime}$. This means that once a photon wave packet of a given circular polarization in the mode with the higher group velocity overtakes that of the slower mode, the system acquires the phase shift $\varphi_{\delta L}=$ $-\chi_{\delta L} l / 2 \delta v$ which depends on polarizations of the photons. For the linear combination of different polarizations in each mode, the transformation is multiplication $\Phi_{i, j}(t \rightarrow \infty)=U_{i, j}^{k, l} \Phi_{k, l}(t \rightarrow-\infty)$ of the amplitudes $\left(\Phi_{-1,-1}, \Phi_{1,-1}, \Phi_{-1,1}, \Phi_{1,1}\right)$ by the matrix

$$
\widehat{U}=\left(\begin{array}{cccc}
e^{i \varphi_{-2}} & 0 & 0 & 0 \\
0 & \frac{e^{i \varphi_{0}+1}}{2} & \frac{e^{i \varphi_{0}-1}}{2} & 0 \\
0 & \frac{e^{i \varphi_{0}}-1}{2} & \frac{e^{i \varphi_{0}}+1}{2} & 0 \\
0 & 0 & 0 & e^{i \varphi_{2}}
\end{array}\right) .
$$

Numbers are the most fascinating result of the consideration performed. For the parameters specified in the caption of Fig 2, and the detuning $\delta \omega=0.033 \omega$, one obtains phases in the matrix Eq.(24): $\varphi_{-2} \simeq \pi / 2$, $\varphi_{2} \simeq-\pi / 2, \varphi_{0}<\pi / 40$. This means that $\widehat{U}$ is pretty close to a one of standard quantum logic gates realized on the photon polarization variables. From the conditions that the photon wave packets of a length $\Delta l \sim 1 \mathrm{~mm}$ interact during the time interval $\Delta l / \delta v$, and that this time interval should be shorter than time of flight $l / v$, one finds the required tube length $l \gtrsim 45 \mathrm{~mm}$. This quantity can be set to the limit of a few photon pules length $\Delta l$ by the parameter optimization.

Concluding, one can conjecture that the strong chiral optical nonlinearity is a common property of the transparent superconductor tubes in magnetic fields that have the parameters close to the dependence suggested by Eq.(20). However, the question of what kind of material can practically be employed for this purpose is open. The answer implies exploration of the optical absorption spectra of all known superconducting substances that, moreover, allow deposition at a supporting transparent surface as a pure homogeneous layer. It also implies first principle calculations if the exploration will not yield a suitable result.

I am deeply grateful to Andrey Varlamov for the discussion and his comments. 


\section{APPENDIX}

In fact, $\frac{e}{m c} \widehat{\vec{p}} \widehat{\vec{A}} \frac{1}{\hbar \omega_{k}} \frac{e}{m c} \widehat{\vec{p}} \widehat{\vec{A}} \sim \frac{\langle\widehat{p}\rangle^{2}}{\hbar \omega_{k}}\left(\frac{e}{m c}\right)^{2} \widehat{\vec{A}}^{2}$, where $\langle\widehat{p}\rangle$ is a typical transition matrix element of the momentum. The matrix element value is of the order of the Fermi momentum $p_{F}$. However, it differs from zero if the initial and the final states of the electron differ in the momentum by the momentum of the virtually absorbed photon $k \hbar$. Moreover, the initial state should belong to the occupied electronic states of the Fermi distribution, and the final - to the empty states. The width of the energy slab which satisfies the latter condition is $\delta E \sim \frac{p_{F} \hbar k}{m}$, and the relative fraction of the slab in the phase space is $\delta E \frac{d \ln \frac{4}{3} \pi p_{F}^{3}}{d \frac{1}{2 m} p_{F}^{2}}$ One therefore arrives at $\langle\widehat{\vec{p}}\rangle^{2} \sim \frac{1}{3}\langle\widehat{p}\rangle^{2} \sim \frac{1}{3} p_{F}^{2} \times \frac{p_{F} \hbar k}{m} \frac{d \ln \frac{4}{3} \pi p_{F}^{3}}{d \frac{1}{2 m} p_{F}^{2}} \sim \frac{1}{3} p_{F}^{2} \times 2 \frac{p_{F} \hbar k}{2 p_{F}} \frac{d \ln \frac{4}{3} \pi p_{F}^{3}}{d p_{F}}$ $\sim p_{F}^{2} \times \frac{\hbar k}{p_{F}} \sim p_{F} \hbar k$ and hence $\frac{\langle\widehat{\hat{p}}\rangle^{2}}{\hbar \omega_{k}}\left(\frac{e}{m c}\right)^{2} \widehat{\vec{A}}^{2} \sim \frac{p_{F} k}{m \omega_{k}} \frac{e^{2}}{m c^{2}} \widehat{\vec{A}}^{2} \sim \frac{v_{F}}{c} \frac{e^{2}}{m c^{2}} \widehat{\vec{A}}^{2}$.

\section{APPENDIX}

With the help of the anticommutation relations for the field operators the Hamiltonian is going to be set to the form consistent with Eq.(7). In the momentum representation for the uniform static vector potential $\vec{A}_{s t}$

$$
\begin{aligned}
\widehat{H} & =\int d k\left\{\omega_{k}\left(\widehat{a}_{k}^{+} \widehat{a}_{k}+\frac{1}{2}\right)+\frac{1}{g} \widehat{\Delta}^{\dagger}(k) \widehat{\Delta}(k)+\frac{1}{2} \widehat{\psi}_{+}^{\dagger}(k)\left(k-\vec{A}_{s t} / c\right)^{2} \widehat{\psi}_{+}(k)+\frac{1}{2} \widehat{\psi}_{-}^{\dagger}(k)\left(k-\vec{A}_{s t} / c\right)^{2} \widehat{\psi}_{-}(k)\right. \\
& +\int d k^{\prime}\left[-\frac{1}{2} \widehat{\Delta}\left(k-k^{\prime}\right)\left(\widehat{\psi}_{+}^{\dagger}(k) \widehat{\psi}_{-}^{\dagger}\left(-k^{\prime}\right)-\widehat{\psi}_{-}^{\dagger}(k) \widehat{\psi}_{+}^{\dagger}\left(-k^{\prime}\right)\right)-\frac{1}{2} \widehat{\Delta}^{\dagger}\left(k-k^{\prime}\right)\left(\widehat{\psi}_{+}(k) \widehat{\psi}_{-}\left(-k^{\prime}\right)-\widehat{\psi}_{-}(k) \widehat{\psi}_{+}\left(-k^{\prime}\right)\right)\right. \\
& \left.\left.+\int d k^{\prime \prime} d k^{\prime \prime \prime} \delta\left(k-k^{\prime}-k^{\prime \prime}+k^{\prime \prime \prime}\right) \frac{\widehat{a}_{k^{\prime \prime}} \widehat{a}_{k^{\prime \prime \prime}}^{+} \vec{u}_{k^{\prime \prime}}^{*} \vec{u}_{k^{\prime \prime \prime}}+h . c .}{\sqrt{\omega_{k^{\prime \prime \prime}} \omega_{k^{\prime \prime}}}}\left(\frac{\pi v}{2 c} \widehat{\psi}_{+}^{\dagger}(k) \widehat{\psi}_{+}\left(k^{\prime}\right)+\frac{\pi v}{2 c} \widehat{\psi}_{-}^{\dagger}(k) \widehat{\psi}_{-}\left(k^{\prime}\right)\right)\right]\right\} .
\end{aligned}
$$

The anticommutation yields

$$
\begin{aligned}
\widehat{H} & =\int d k\left\{\omega_{k}\left(\widehat{a}_{k}^{+} \widehat{a}_{k}+\frac{1}{2}\right)+\frac{1}{g} \widehat{\Delta}^{\dagger}(k) \widehat{\Delta}(k)+\frac{1}{2} \widehat{\psi}_{+}^{\dagger}(k)\left(k-\vec{A}_{s t} / c\right)^{2} \widehat{\psi}_{+}(k)+\frac{1}{2} \widehat{\psi}_{-}^{\dagger}(k)\left(k-\vec{A}_{s t} / c\right)^{2} \widehat{\psi}_{-}(k)\right. \\
& +\int d k^{\prime}\left[-\frac{1}{2}\left(\widehat{\Delta}\left(k-k^{\prime}\right) \widehat{\psi}_{+}^{\dagger}(k) \widehat{\psi}_{-}^{\dagger}\left(-k^{\prime}\right)-\widehat{\Delta}\left(k-k^{\prime}\right) \widehat{\psi}_{+}^{\dagger}\left(-k^{\prime}\right) \widehat{\psi}_{-}^{\dagger}(k)\right)\right. \\
& -\frac{1}{2}\left(-\widehat{\Delta}^{\dagger}\left(k-k^{\prime}\right) \widehat{\psi}_{-}\left(-k^{\prime}\right) \widehat{\psi}_{+}(k)+\widehat{\Delta}^{\dagger}\left(k-k^{\prime}\right) \widehat{\psi}_{-}(k) \widehat{\psi}_{+}\left(-k^{\prime}\right)\right) \\
& \left.\left.+\int d k^{\prime \prime} d k^{\prime \prime \prime} \delta\left(k-k^{\prime}-k^{\prime \prime}+k^{\prime \prime \prime}\right) \frac{\widehat{a}_{k^{\prime \prime}} \widehat{a}_{k^{\prime \prime \prime}}^{+} \vec{u}_{k^{\prime \prime}}^{*} \vec{u}_{k^{\prime \prime \prime}}+h . c .}{\sqrt{\omega_{k^{\prime \prime \prime}} \omega_{k^{\prime \prime}}}}\left(\frac{\pi v}{2 c} \widehat{\psi}_{+}^{\dagger}(k) \widehat{\psi}_{+}\left(k^{\prime}\right)-\frac{\pi v}{2 c} \widehat{\psi}_{-}\left(k^{\prime}\right) \widehat{\psi}_{-}^{\dagger}(k)\right)\right]\right\} .
\end{aligned}
$$

Now one changes the integration variables $k \rightarrow \bar{k}, k^{\prime} \rightarrow \bar{k}^{\prime}$ in two last components of the vector

$$
\begin{aligned}
\widehat{H} & =\int d k\left\{\omega_{k}\left(\widehat{a}_{k}^{+} \widehat{a}_{k}+\frac{1}{2}\right)+\frac{1}{g} \widehat{\Delta}^{\dagger}(k) \widehat{\Delta}(k)+\frac{1}{2} \widehat{\psi}_{+}^{\dagger}(k)\left(k-\vec{A}_{s t} / c\right)^{2} \widehat{\psi}_{+}(k)+\frac{1}{2} \widehat{\psi}_{-}^{\dagger}(k)\left(k-\vec{A}_{s t} / c\right)^{2} \widehat{\psi}_{-}(k)\right. \\
& +\int d k^{\prime}\left[-\frac{1}{2}\left(\widehat{\Delta}\left(k-\bar{k}^{\prime}\right) \widehat{\psi}_{+}^{\dagger}(k) \widehat{\psi}_{-}^{\dagger}\left(-\bar{k}^{\prime}\right)+\widehat{\Delta}\left(k^{\prime}-\bar{k}\right) \widehat{\psi}_{+}^{\dagger}\left(k^{\prime}\right) \widehat{\psi}_{-}^{\dagger}(-\bar{k})\right)\right. \\
& -\frac{1}{2}\left(-\widehat{\Delta}^{\dagger}\left(k-\bar{k}^{\prime}\right) \widehat{\psi}_{-}\left(-\bar{k}^{\prime}\right) \widehat{\psi}_{+}(k)-\widehat{\Delta}^{\dagger}\left(k^{\prime}-\bar{k}_{)} \widehat{\psi}_{-}(-\bar{k}) \widehat{\psi}_{+}\left(k^{\prime}\right)\right)\right. \\
& \left.\left.+\int d k^{\prime \prime} d k^{\prime \prime \prime} \delta\left(k-k^{\prime}-k^{\prime \prime}+k^{\prime \prime \prime}\right) \frac{\widehat{a}_{k^{\prime \prime}} \widehat{a}_{k^{\prime \prime \prime}}^{+} \vec{u}_{k^{\prime \prime}}^{*} \vec{u}_{k^{\prime \prime \prime}}+h \cdot c .}{\sqrt{\omega_{k^{\prime \prime \prime}} \omega_{k^{\prime \prime}}}}\left(\frac{\pi v}{2 c} \widehat{\psi}_{+}^{\dagger}(k) \widehat{\psi}_{+}\left(k^{\prime}\right)-\frac{\pi v}{2 c} \widehat{\psi}_{-}\left(k^{\prime}\right) \widehat{\psi}_{-}^{\dagger}(k)\right)\right]\right\},
\end{aligned}
$$

and arrives to the matrix form

$$
\begin{aligned}
& \left(\widehat{\psi}_{+}^{\dagger}\left(k^{\prime}\right) \widehat{\psi}_{+}^{\dagger}(k) \widehat{\psi}_{-}(-\bar{k}) \widehat{\psi}_{-}\left(-\bar{k}^{\prime}\right)\right) \times \\
& \left(\begin{array}{cccc}
\frac{1}{2}\left(k^{\prime}-\vec{A}_{s t} / c\right)^{2} & \frac{\pi v}{2 c} \frac{\vec{u}_{k^{\prime \prime}}^{* \prime} \vec{u}_{k^{\prime \prime \prime}} \widehat{a}_{k^{\prime \prime}} \widehat{a}_{k^{\prime \prime \prime}}^{+}}{\sqrt{\omega_{k^{\prime \prime \prime}} \omega_{k^{\prime \prime}}}} & \frac{1}{2} \widehat{\Delta}\left(k^{\prime}-\bar{k}\right) & \frac{1}{2} \widehat{\Delta}(S) \\
\frac{\pi v}{2 c} \frac{\vec{u}_{k^{\prime \prime}} \vec{u}_{k^{\prime \prime \prime}}^{* \prime \prime} \widehat{a}_{k^{\prime \prime}}^{+} \widehat{a}_{k^{\prime \prime \prime}}}{\sqrt{\omega_{k^{\prime \prime \prime}} \omega_{k^{\prime \prime}}}} & \frac{1}{2}\left(k-\vec{A}_{s t} / c\right)^{2} & \frac{1}{2} \widehat{\Delta}(S) & \frac{1}{2} \widehat{\Delta}\left(k-\bar{k}^{\prime}\right) \\
\frac{1}{2} \widehat{\Delta}^{\dagger}\left(k^{\prime}-\bar{k}\right) & \frac{1}{2} \widehat{\Delta}^{\dagger}(S) & -\frac{1}{2}\left(-\bar{k}-\vec{A}_{s t} / c\right)^{2} & -\frac{\pi v}{2 c} \frac{\vec{u}_{k^{\prime \prime}} \vec{u}_{k^{\prime \prime \prime}}^{* \prime \prime} \widehat{a}_{k^{\prime \prime}}^{+} \widehat{a}_{k^{\prime \prime \prime}}}{\sqrt{\omega_{k^{\prime \prime \prime}} \omega_{k^{\prime \prime}}}} \\
\frac{1}{2} \widehat{\Delta}^{\dagger}(S) & \frac{1}{2} \widehat{\Delta}^{\dagger}\left(k-\bar{k}^{\prime}\right) & -\frac{\pi v}{2 c} \frac{\vec{u}_{k^{\prime \prime}}^{*} \vec{u}_{k^{\prime \prime \prime}} \widehat{a}_{k^{\prime \prime}} \widehat{a}_{k^{\prime \prime \prime}}^{+\prime \prime}}{\sqrt{\omega_{k^{\prime \prime \prime}} \omega_{k^{\prime \prime}}}}-\frac{1}{2}\left(-\bar{k}^{\prime}-\vec{A}_{s t} / c\right)^{2}
\end{array}\right)\left(\begin{array}{c}
\widehat{\psi}_{+}\left(k^{\prime}\right) \\
\widehat{\psi}_{+}(k) \\
\widehat{\psi}_{-}^{\dagger}(-\bar{k}) \\
\widehat{\psi}_{-}^{\dagger}\left(-\bar{k}^{\prime \prime}\right)
\end{array}\right)
\end{aligned}
$$


for the fermionic part. Now the replacement $\bar{k}^{\prime} \rightarrow k^{\prime}+S, \bar{k} \rightarrow k+S$

$$
\begin{aligned}
& \omega_{k}\left(\widehat{a}_{k}^{+} \widehat{a}_{k}+\frac{1}{2}\right)-\frac{1}{2 g} \widehat{\Delta}^{\dagger}(\vec{k}) \widehat{\Delta}(\vec{k})+\left(\widehat{\psi}_{+}^{\dagger}\left(k^{\prime}\right) \widehat{\psi}_{+}^{\dagger}(k) \widehat{\psi}_{-}(-k-S) \widehat{\psi}_{-}\left(-k^{\prime}-S\right)\right) \times
\end{aligned}
$$

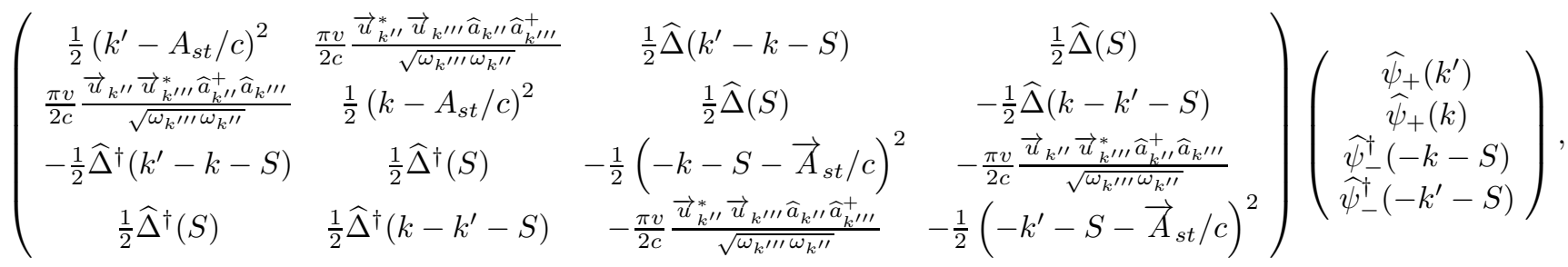

where $S$ denotes the momentum shift in the presence of a magnetic field.

For the photon with the wavenumber difference $k^{\prime \prime \prime}-k^{\prime \prime} \rightarrow \delta k$, make replacement in the electron arguments $k \rightarrow \widetilde{k}$, $k^{\prime} \rightarrow \widetilde{k}+\delta k$ and the photon arguments $k^{\prime \prime \prime} \rightarrow k^{\prime} \rightarrow k+\delta k, k^{\prime \prime} \rightarrow k$, then the integrand adopts the form

$$
\begin{aligned}
& \frac{1}{2 g} \widehat{\Delta}^{\dagger}(\delta k-S) \widehat{\Delta}(\delta k-S)+\frac{1}{2 g} \widehat{\Delta}^{\dagger}(-\delta k-S) \widehat{\Delta}(-\delta k-S)+\omega_{k}\left(\widehat{a}_{k}^{+} \widehat{a}_{k}+\frac{1}{2}\right)+\omega_{k+\delta k}\left(\widehat{a}_{k+\delta k}^{+} \widehat{a}_{k+\delta k}+\frac{1}{2}\right) \\
& +\frac{1}{2}\left(\widehat{\psi}_{+}^{\dagger}(\widetilde{k}+\delta k) \widehat{\psi}_{+}^{\dagger}(\widetilde{k}) \widehat{\psi}_{-+}(-\widetilde{k}-S) \widehat{\psi}_{-+}(-\widetilde{k}-\delta k-S)\right) \times \\
& \left(\begin{array}{cccc}
\left(\widetilde{k}+\delta k-\frac{A_{s t}}{c}\right)^{2} & \frac{\pi v}{c} \frac{\vec{u}_{k}^{*} \vec{u}_{k+\delta k} \widehat{a}_{k} \widehat{a}_{k+\delta k}^{+}}{\sqrt{\omega_{k+\delta k} \omega_{k}}} & \widehat{\Delta}(\delta k-S) & \widehat{\Delta}(S) \\
\frac{\pi v}{c} \frac{\vec{u}_{k} \vec{u}_{k+\delta k}^{*} \widehat{a}_{k}^{+} \widehat{a}_{k+\delta k}}{\sqrt{\omega_{k+\delta k} \omega_{k}}} & \left(\widetilde{k}-\frac{A_{s t}}{c}\right)^{2} & \widehat{\Delta}(S) & \widehat{\Delta}(-\delta k-S) \\
\widehat{\Delta}^{\dagger}(\delta k-S) & \widehat{\Delta}^{\dagger}(S) & -\left(-\widetilde{k}-S-\frac{A_{s t}}{c}\right)^{2} & -\frac{\pi v}{c} \frac{\vec{u}_{k} \vec{u}_{k+\delta k}^{*} \widehat{a}_{k}^{+} \widehat{a}_{k+\delta k}}{\sqrt{\omega_{k+\delta k} \omega_{k}}} \\
\widehat{\Delta}^{\dagger}(S) & \widehat{\Delta}^{\dagger}(-\delta k-S) & -\frac{\pi v}{c} \frac{\vec{u}_{k}^{*} \vec{u}_{k+\delta k} \widehat{a}_{k} \widehat{a}_{k+\delta k}^{+}}{\sqrt{\omega k+\delta k \omega_{k}}}-\left(-\widetilde{k}-\delta k-S-\frac{A_{s t}}{c}\right)^{2}
\end{array}\right)\left(\begin{array}{c}
\widehat{\psi}_{+}(\widetilde{k}+\delta k) \\
\widehat{\psi}_{+}(\widetilde{k}) \\
\widehat{\psi}_{-}^{\dagger}(-\widetilde{k}-S) \\
\widehat{\psi}_{-}^{\dagger}(-\widetilde{k}-\delta k-S)
\end{array}\right) .
\end{aligned}
$$

Before the cooling, the magnetic field potential is given as $\frac{A_{s t}}{c} \rightarrow \Lambda$, and after the cooling the coupling occur among the electron states with the same energy $(\widetilde{k}-\Lambda)^{2}=(-\widetilde{k}-S-\Lambda)^{2}$, hence $S=-2 \Lambda$. One therefore has

$$
\begin{aligned}
& \frac{1}{2 g} \widehat{\Delta}^{\dagger}(\delta k-S) \widehat{\Delta}(\delta k-S)+\frac{1}{2 g} \widehat{\Delta}^{\dagger}(-\delta k-S) \widehat{\Delta}(-\delta k-S)+\omega_{k}\left(\widehat{a}_{k}^{+} \widehat{a}_{k}+\frac{1}{2}\right)+\omega_{k+\delta k}\left(\widehat{a}_{k+\delta k}^{+} \widehat{a}_{k+\delta k}+\frac{1}{2}\right) \\
& +\frac{1}{2}\left(\widehat{\psi}_{+}^{\dagger}(\widetilde{k}+\delta k) \widehat{\psi}_{+}^{\dagger}(\widetilde{k}) \widehat{\psi}_{-}(-\widetilde{k}-S) \widehat{\psi}_{-}(-\widetilde{k}-\delta k-S)\right) \times \\
& \left(\begin{array}{cccc}
\left(\widetilde{k}+\delta k-\frac{A_{s t}}{c}\right)^{2} & \frac{\pi v}{c} \frac{\vec{u}_{k}^{*} \vec{u}_{k+\delta k} \widehat{a}_{k} \widehat{a}_{k+\delta k}^{+}}{\sqrt{\omega_{k+\delta k} \omega_{k}}} & \widehat{\Delta}(\delta k+2 \Lambda) & \widehat{\Delta}(-2 \Lambda) \\
\frac{\pi v}{c} \frac{\vec{u}_{k} \vec{u}_{k+\delta k}^{*} \widehat{a}_{k}^{+} \widehat{a}_{k+\delta k}}{\sqrt{\omega_{k+\delta k} \omega_{k}}} & \left(\widetilde{k}-\frac{A_{s t}}{c}\right)^{2} & \widehat{\Delta}(-2 \Lambda) & \widehat{\Delta}(-\delta k+2 \Lambda) \\
\widehat{\Delta}^{\dagger}(\delta k+2 \Lambda) & \widehat{\Delta}^{\dagger}(-2 \Lambda) & -\left(-\widetilde{k}+2 \Lambda-\frac{A_{s t}}{c}\right)^{2} & -\frac{\pi v}{c} \frac{\vec{u}_{k} \vec{u}_{k+\delta k}^{*} \widehat{a}_{k}^{+} \widehat{a}_{k+\delta k}}{\sqrt{\omega_{k+\delta k} \omega_{k}}} \\
\widehat{\Delta}^{\dagger}(-2 \Lambda) & \widehat{\Delta}^{\dagger}(-\delta k+2 \Lambda) & -\frac{\pi v}{c} \frac{\vec{u}_{k}^{*} \vec{u}_{k+\delta k} \widehat{a}_{k} \widehat{a}_{k+\delta k}^{+}}{\sqrt{\omega k+\delta k \omega_{k}}} & -\left(-\widetilde{k}-\delta k+2 \Lambda-\frac{A_{s t}}{c}\right)^{2}
\end{array}\right)\left(\begin{array}{c}
\widehat{\psi}_{+}(\widetilde{k}+\delta k) \\
\widehat{\psi}_{+}(\widetilde{k}) \\
\widehat{\psi}_{-}^{\dagger}(-\widetilde{k}+2 \Lambda) \\
\widehat{\psi}_{-}^{\dagger}(-\widetilde{k}-\delta k+2 \Lambda)
\end{array}\right)
\end{aligned}
$$

This expression implies that after the cooling the magnetic field has been changed and now it is given by the vector potential $\frac{A_{s t}}{c}$, which is different from $\Lambda$. To avoid confusion note, that later on, for the case of the cylindric setting, the field vector potential $A_{s t}$ will be parametrized by the number of the magnetic field quanta traversing the cylinder cross-section and will be treated as an angular momentum.

Also note, that the main role of the "frozen" part of the magnetic potential $\Lambda$ is to avoid interference $\widehat{\Delta}(\delta k+2 \Lambda)$ and $\widehat{\Delta}(-\delta k+2 \Lambda)$, since otherwise, $\widehat{\Delta}^{+}(\delta k)=\widehat{\Delta}(-\delta k)$, and the cross couplings of the Cooper pair's electrons before and after the virtual transition may, and do cancel the momentum sensitive part of the nonlinear coupling.

The matrix Eq.(8) comes from the electron part of the action Eq.(7) with $\widehat{M}=i \partial_{t}-\widehat{H}$, where the Hamiltonian corresponds to the electron part of Eq.(25). The time derivative part experience no transformation when the electron field operators are interchanged, since sign change due to the change of the order of the fermionic operators is followed by transferring of the time derivative operator from the left field operator $\psi$ to the right one, and hence in the Fourier 
representation

$$
i \partial_{t} \rightarrow\left(\begin{array}{cccc}
\delta \omega+\widetilde{\omega} & 0 & 0 & 0 \\
0 & \widetilde{\omega} & 0 & 0 \\
0 & 0 & \widetilde{\omega} & 0 \\
0 & 0 & 0 & \delta \omega+\widetilde{\omega}
\end{array}\right)
$$

which only allows for the energy shift $\delta \omega$ of the Cooper pair after virtual absorption of the photon: before the absorption - positions 2 and 3, and after the absorption - positions 1 and 4. This form is consistent with Eq.(4.5) of [9]

\section{APPENDIX}

The relations between the magnetic field and the vector potential components in cylindrical coordinates read

$$
\begin{aligned}
B_{r} & =\frac{1}{r} \frac{\partial}{\partial \theta} A_{z}-\frac{\partial}{\partial z} A_{\theta}, \\
B_{\theta} & =\frac{\partial}{\partial z} A_{r}-\frac{\partial}{\partial r} A_{z}, \\
B_{z} & =\frac{1}{r} \frac{\partial}{\partial r}\left(r A_{\theta}\right)-\frac{\partial}{r \partial \theta} A_{r} .
\end{aligned}
$$

Outside the tube the fields satisfying the wave equation are

$$
\begin{aligned}
A_{r} & =\left[\frac{i}{2} \bar{D} \frac{m}{r q} K_{m}(r q)-\frac{i}{2} D K_{m}^{\prime}(r q)\right] e^{i(\omega t-k z-m \theta)} \\
A_{\theta} & =\left[\frac{1}{2} D \frac{m}{r q} K_{m}(r q)-\frac{1}{2} \bar{D} K_{m}^{\prime}(r q)\right] e^{i(\omega t-k z-m \theta)} \\
A_{z} & =\frac{-q}{2 k} D K_{m}(r q) e^{i(\omega t-k z-m \theta)} \\
B_{r} & =\left[\frac{i}{2} D \frac{\omega^{2}}{k} \frac{m}{r q} K_{m}(r q)-\frac{i}{2} \bar{D} k K_{m}^{\prime}(r q)\right] e^{i(\omega t-k z-m \theta)} \\
B_{\theta} & =\left[\frac{1}{2} \bar{D} \frac{k m}{r q} K_{m}(r q)-\frac{1}{2} \frac{\omega^{2}}{k} D K_{m}^{\prime}(r q)\right] e^{i(\omega t-k z-m \theta)} \\
B_{z} & =-\frac{q}{2} \bar{D} K_{m}(r q) e^{i(\omega t-k z-m \theta)}
\end{aligned}
$$

where $q=\sqrt{k^{2}-\left(\frac{\omega}{c}\right)^{2}}, K_{m}(x)$ are the modified Bessel function regular at $x \rightarrow \infty$, and $D, \bar{D}$ are the constants to be determined. Inside the tube, for the radial parts multiplying the phase factor $e^{i(\omega t-k z-m \theta)}$ one takes

$$
\begin{aligned}
A_{r} & =\frac{i}{2} \bar{A} \frac{m}{r q} I_{m}(r q)-\frac{i}{2} A I_{m}^{\prime}(r q) \\
A_{\theta} & =\frac{1}{2} A \frac{m}{r q} I_{m}(r q)-\frac{1}{2} \bar{A} I_{m}^{\prime}(r q) \\
A_{z} & =\frac{-q}{2 k} A I_{m}(r q) \\
B_{r} & =\frac{i}{2} A \frac{\omega^{2}}{k} \frac{m}{r q} I_{m}(r q)-\frac{i}{2} \bar{A} k I_{m}^{\prime}(r q) \\
B_{\theta} & =\frac{1}{2} \bar{A} \frac{k m}{r q} I_{m}(r q)-\frac{1}{2} A \frac{\omega^{2}}{k} I_{m}^{\prime}(r q) \\
B_{z} & =-\frac{q}{2} \bar{A} I_{m}(r q),
\end{aligned}
$$


where the constants are $A$ and $\bar{A}$, and the modified Bessel functions $I_{m}(r q)$ are regular at $x=0$. Within the walls of the tube, for the radial parts one finds

$$
\begin{aligned}
A_{r} & =\frac{1}{2}\left(-B \varepsilon Y_{m}^{\prime}(r p)+\bar{B} \varepsilon \frac{m}{r p} Y_{m}(r p)-C \varepsilon J_{m}^{\prime}(r p)+\bar{C} \varepsilon \frac{m}{r p} J_{m}(r p)\right) \\
A_{\theta} & =\frac{1}{2}\left(-\bar{B} Y_{m}^{\prime}(r p)+B \frac{m}{r p} Y_{m}(r p)-\bar{C} J_{m}^{\prime}(r p)+C \frac{m}{r p} J_{m}(r p)\right) \\
A_{z} & =\frac{1}{2} \frac{p}{k} B Y_{m}(r p)+\frac{1}{2} \frac{p}{k} C J_{m}(r p) \\
B_{r} & =\frac{1}{2}\left(-\bar{B} k Y_{m}^{\prime}(r p)+B \frac{n^{2} \omega^{2}}{k c^{2}} \frac{m}{r p} Y_{m}(r p)-\bar{C} k J_{m}^{\prime}(r p)+C \frac{n^{2} \omega^{2}}{k c^{2}} \frac{m}{r p} J_{m}(r p)\right) \\
B_{\theta} & =\frac{1}{2}\left(\bar{B} k \frac{1}{\bar{\mu}} \frac{m}{r p} Y_{m}(r p)-\frac{1}{\mu} \frac{n^{2} \omega^{2}}{k c^{2}} B Y_{m}^{\prime}(r p)+\bar{C} k \frac{1}{\bar{\mu}} \frac{m}{r p} J_{m}(r p)-\frac{1}{\bar{\mu}} \frac{n^{2} \omega^{2}}{k c^{2}} C J_{m}^{\prime}(r p)\right) \\
\frac{1}{\bar{\mu}} B_{z} & =\frac{1}{\bar{\mu}} \frac{1}{2} p \bar{B} Y_{m}(r p)+\frac{1}{\bar{\mu}} \frac{1}{2} p \bar{C} J_{m}(r p),
\end{aligned}
$$

where $J_{m}(x)$ and $Y_{m}(x)$ are the Bessel functions, the corresponding coefficients are $C, \bar{C}$, $B$, and $\bar{B}$, while $p=$ $\sqrt{\left(n \frac{\omega}{c}\right)^{2}-k^{2}}$. Here $n=\sqrt{\bar{\mu} \varepsilon}$ is the refraction index, where $\bar{\mu}$ and $\varepsilon$ are the magnetic and the dielectric linear susceptibilities, respectively.

Conditions of the tangential fields continuity at the inner $R_{\text {inn }}=R_{1}$ and the outer $R_{\text {out }}=R_{2}$ radii of the tube can be written as a product of a vector by matrix

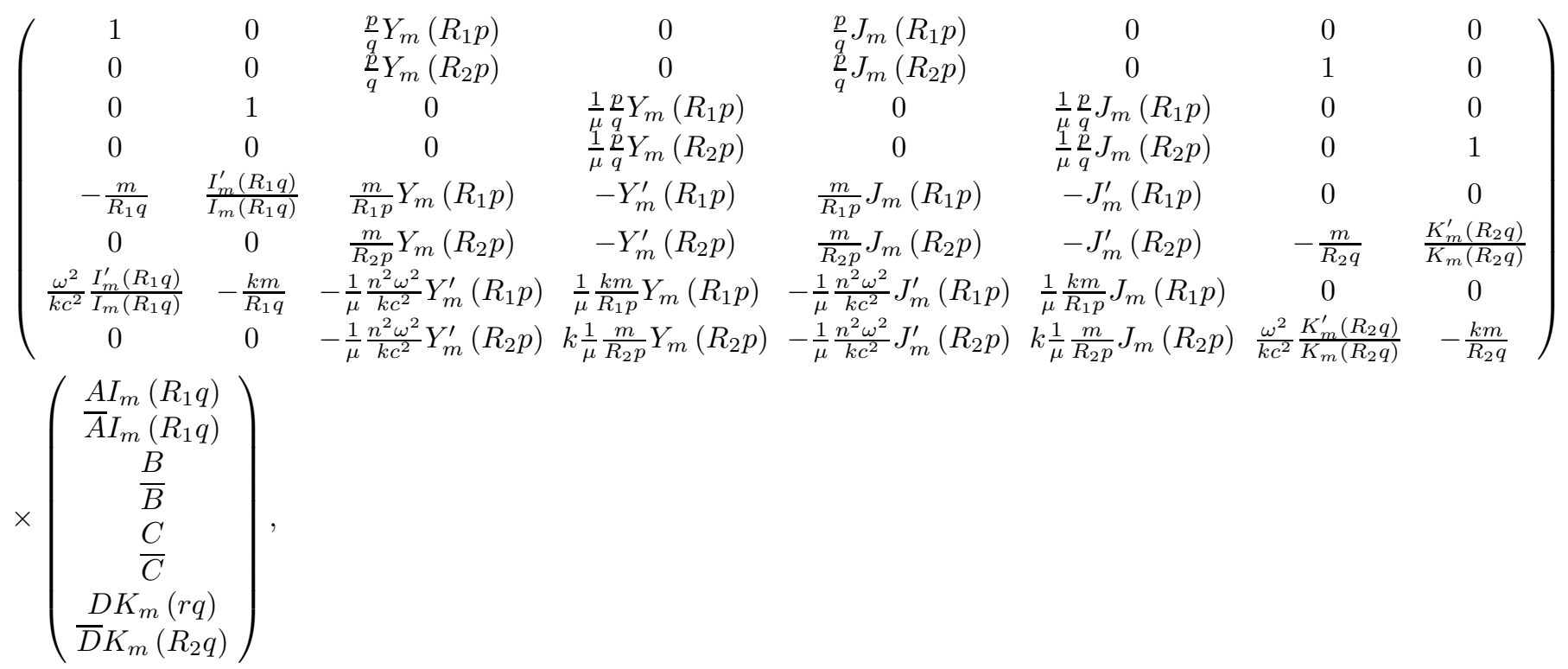

which should give zero vector for nonzero $(A, \bar{A}, \ldots D, \bar{D})$. This implies, that the determinant of the matrix above equals zero, and the vector multiplying this matrix is an eigenvector corresponding to zero eigenvalue. This vector will give the coefficients $A, \ldots, \bar{D}$ and thereby the fields distribution, corresponding to the value of the wavevector $k(\omega)$ making the determinant equal to zero.

In the following figure 


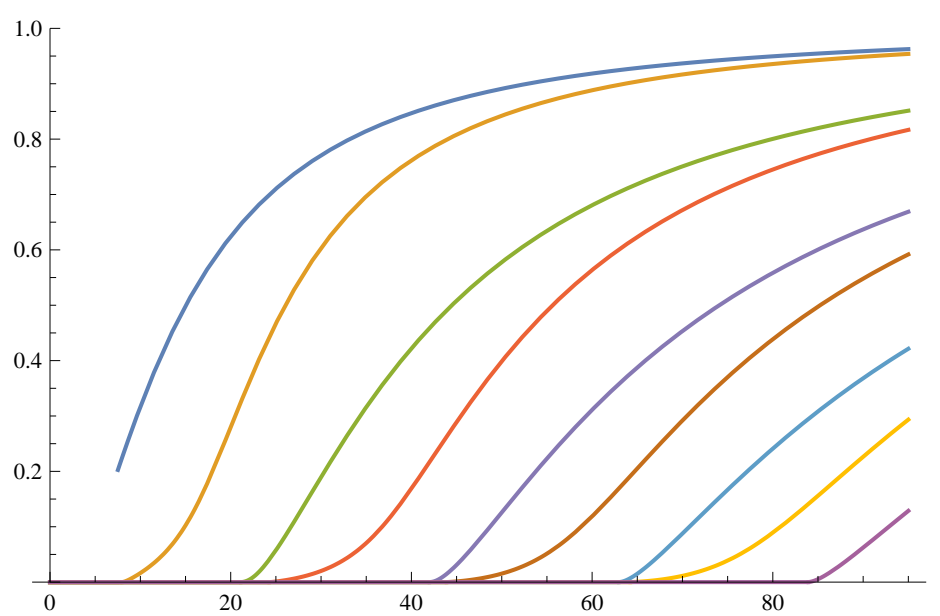

Phase velocities $n=2.26, R_{2} / R_{1}=1.15 . R_{1}=3 \mu, \lambda_{1}=1 \mu, \lambda_{2}=0.9992 \mu$ On the abscise axis - scaled frequency $w=R_{i n n} \frac{\omega}{c} \sqrt{n^{2}-1}$, on the ordinate axis scaled and shifted phase velocity $b=\frac{\frac{k c}{\omega}-1}{n-1}$.

one sees dependences $k(\omega)$ that have been found numerically for the double degenerate modes corresponding $m= \pm 1$. Each of the mode can carry a polarized photon, such that the quantum information can be encoded in the photon polarization. In the following figure

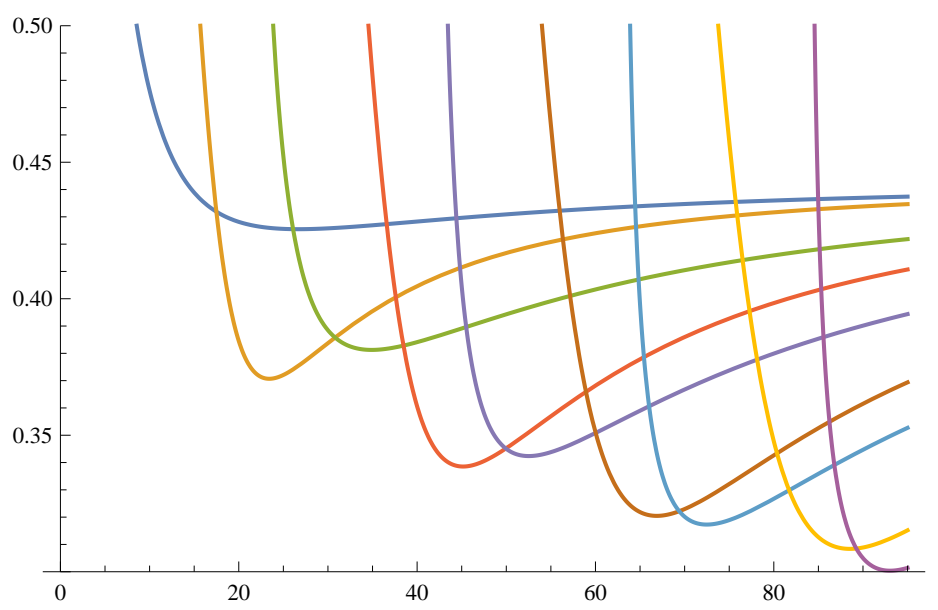

Group velocities $n=2.26$. On the abscise axis - scaled frequency $w=R_{i n n} \frac{w}{c} \sqrt{n^{2}-1}$, on the ordinate axis scaled group velocity $v / c$.

one sees the corresponding group velocities and the frequencies where the group velocities of different modes coincide.

Field distribution for the components of vector potential corresponding to a point close to the point of the group velocity coincidence of the first and the second modes given by the corresponding coefficients are shown in the following figure 


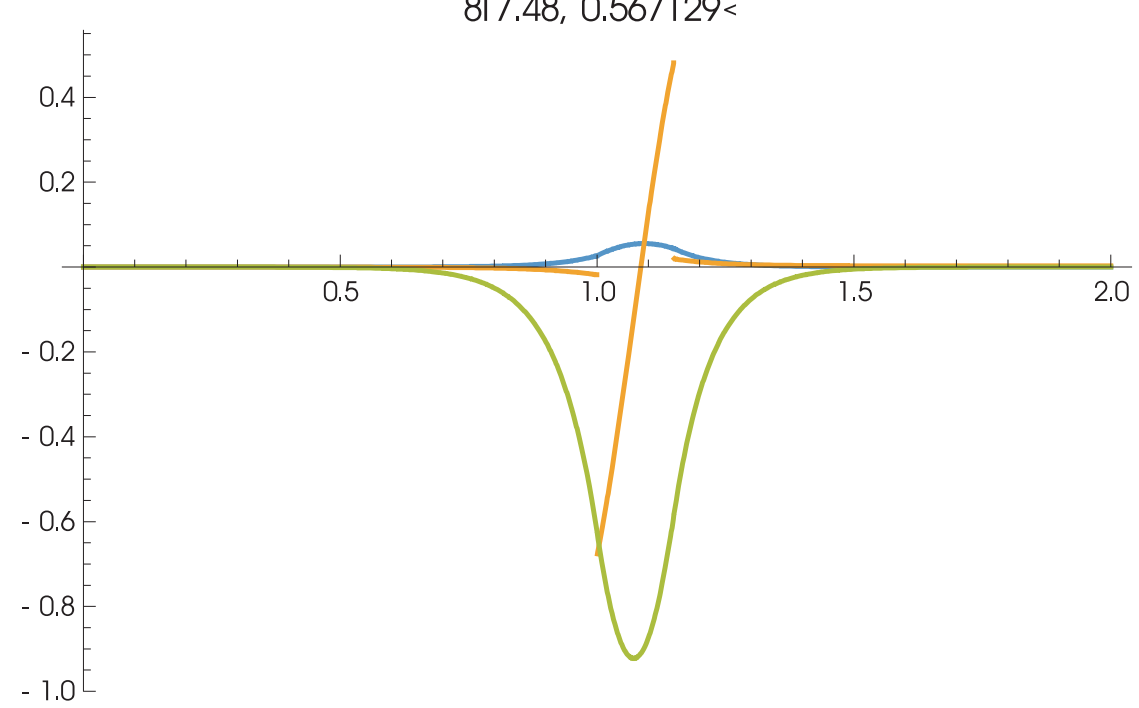

Vector potential components for the first mode: the radial -blue, the azimutal component -brown, and the longitudinal component - green. Numbers at the plot are the coordinates $w$ and $b$ of the Figure for the phase velocity

and the figure

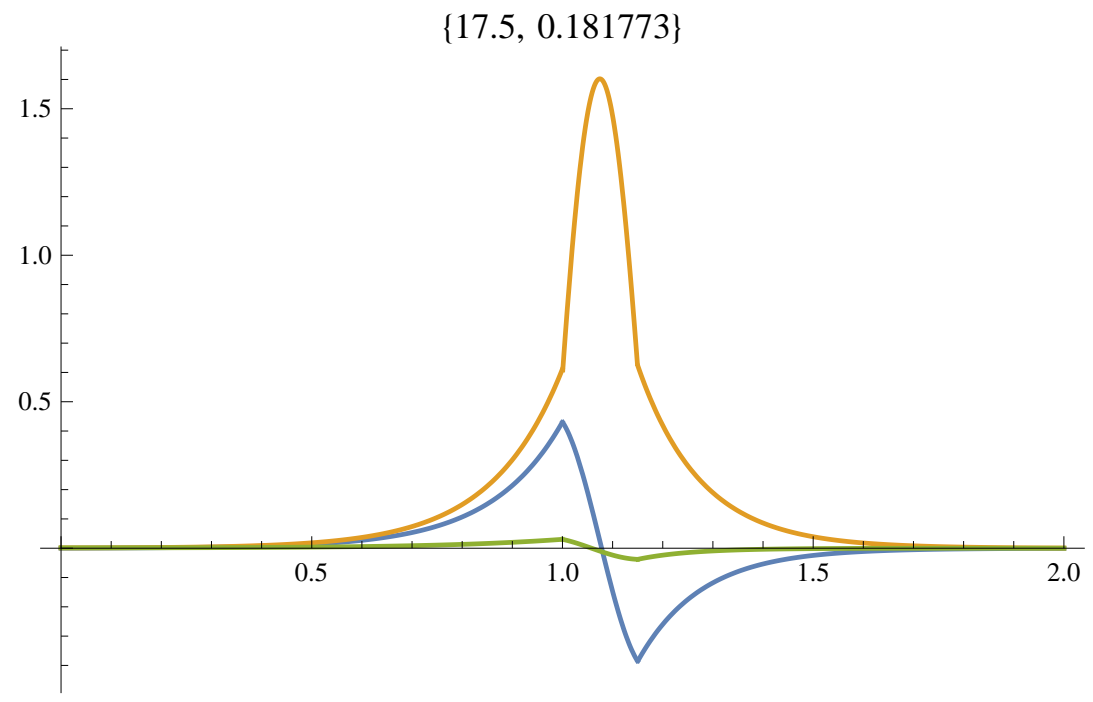

Vector potential components for the second mode: the radial -blue, the azimutal component -brown, and the longitudinal component - green. Numbers at the plot are the coordinates $w$ and $b$ of the Figure for the phase velocity

for the first and the second modes, respectively. The mode fields are normalized by the requirement $\int\left(\vec{u}_{k}^{*}(R) \cdot \vec{u}_{k}(R)\right) R d R=1$

One can equally find the scalar product of the vector potential of the first mode by that of the second one. The following figure 
$817.48,0.567129,17.5,0.181773<$

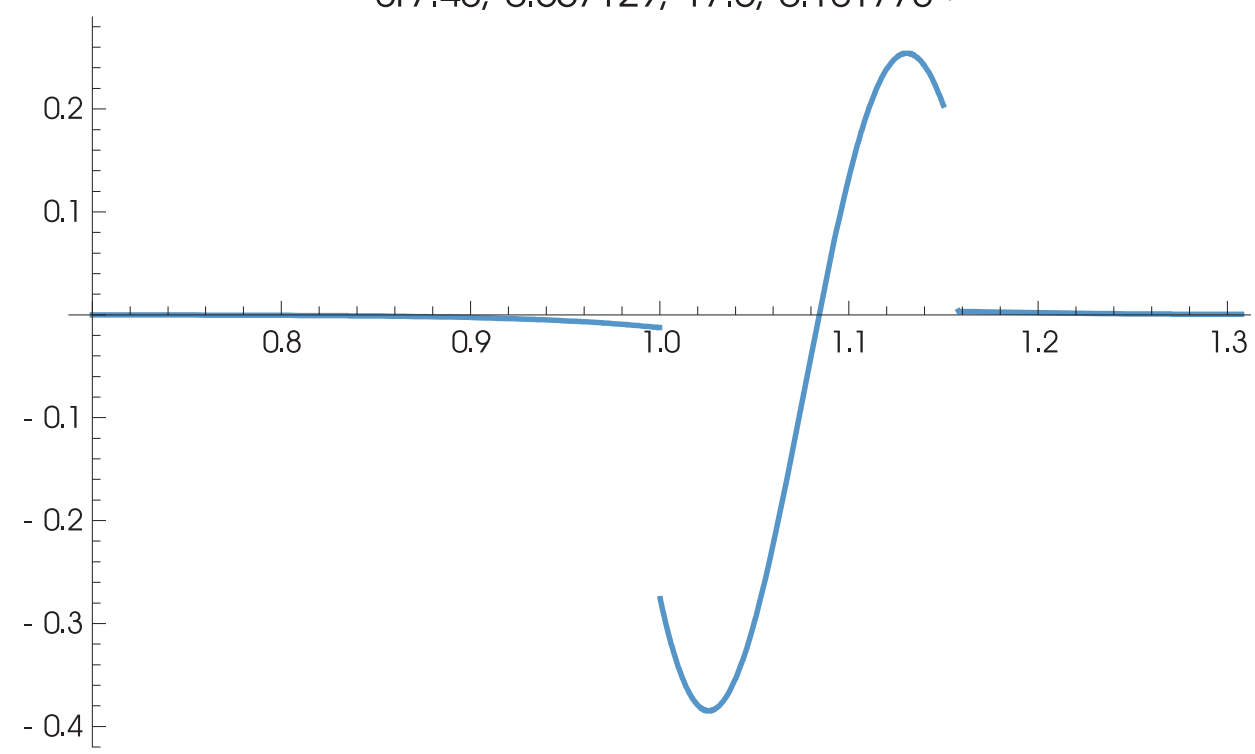

Scalar product of mode vector potentials as a function of the radius. The maximum product -0.4 corresponds to $R=1.05 R_{i n n}$. The frequency difference $\delta \omega$ corresponds to the wavelength difference of $0,96 \mathrm{~mm}$. Numbers at the plot are the coordinates $w$ and $b$ of the Figure for the phase velocity. Normalization $\int\left(\vec{u}_{k}^{*}(R) \cdot \vec{u}_{k^{\prime}}(R)\right) R d R=1$ is done in the dimensionless unities $R_{i n n}=1$.

shows the dependence of the scalar product on the radius. Note that the integral $\int\left(\vec{u}_{k}^{*}(R) \cdot \vec{u}_{k^{\prime}}(R)\right) R d R$ of the scalar product vanishes for equal frequencies $\omega=\omega^{\prime}$. One sees that this is almost the case at the lst figure

\section{APPENDIX E}

One considers the action integrand

$$
L g=\ln \left[\operatorname{det}\left(\begin{array}{cccc}
\delta \omega+\widetilde{\omega}-\epsilon_{1} & \alpha^{*} \vec{u}_{k}^{*} \vec{u}_{k^{\prime}} & -\Delta_{1} & -\Delta \\
\alpha \vec{u}_{k^{\prime}}^{*} \vec{u}_{k} & \widetilde{\omega}-\epsilon_{2} & -\Delta & -\Delta_{2} \\
-\Delta^{*}{ }_{1} & -\Delta^{*} & \widetilde{\omega}+\epsilon_{3} & -\alpha \vec{u}_{k^{\prime}}^{*} \vec{u}_{k} \\
-\Delta^{*} & -\Delta_{2}^{*} & -\alpha^{*} \vec{u}_{k}^{*} \vec{u}_{k^{\prime}} & \delta \omega+\widetilde{\omega}+\epsilon_{4}
\end{array}\right)\right] \text {. }
$$

nd performs the Taylor expansion

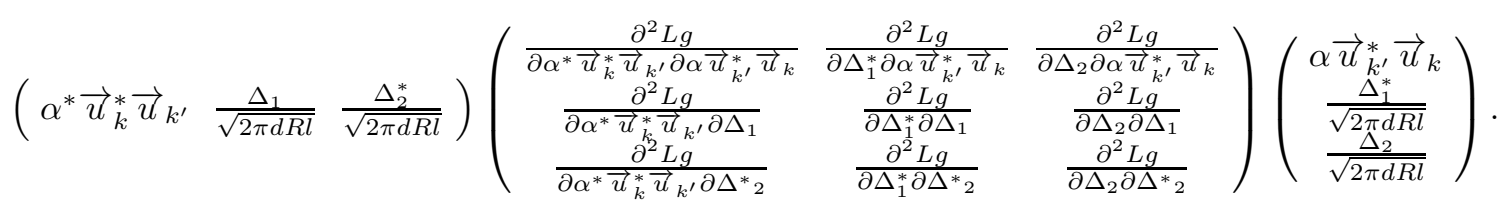

Explicit form of the matrix reads

$$
\begin{aligned}
& \frac{\partial^{2} L g}{\partial \ldots \partial \ldots}=\left(\begin{array}{ccc}
\frac{\partial^{2} L g}{\partial \alpha^{*} \bar{u}_{k}^{*} \bar{u}_{k^{\prime}} \partial \alpha \bar{u}_{k^{\prime}}^{*} \bar{u}_{k}} & \frac{\partial^{2} L g}{\partial \Delta_{1}^{*} \partial \alpha \bar{u}^{*}, \bar{u}_{k}} & \frac{\partial^{2} L g}{\partial \Delta_{2} \partial \alpha \bar{u}^{*} \bar{u}_{k}} \\
\frac{\partial^{2} L g}{\partial \alpha^{*} \bar{u}_{k}^{*} \bar{u}_{k^{\prime}} \partial \Delta_{1}} & \frac{\partial^{2} L g}{\partial \Delta_{1}^{*} \partial \Delta_{1}} & \frac{\partial^{2} L g}{\partial \Delta_{2} \partial \Delta_{1}} \\
\frac{\partial^{2} L g}{\partial \alpha^{*} \bar{u}_{k}^{*} \bar{u}_{k^{\prime}} \partial \Delta^{*}{ }_{2}} & \frac{\partial^{2} L g}{\partial \Delta_{1}^{*} \partial \Delta^{*}} & \frac{\partial^{2} L g}{\partial \Delta_{2} \partial \Delta^{*}}
\end{array}\right) \\
& \equiv\left(\begin{array}{ccc}
L g_{\alpha, \alpha} & \frac{\Delta}{|\Delta|} L g_{\Delta_{1}, \alpha} & \frac{\Delta^{*}}{|\Delta|} L g_{\Delta_{2}, \alpha} \\
\frac{\Delta^{*}}{|\Delta|} L g_{\Delta_{1}, \alpha} & L g_{\Delta_{1}, \Delta_{1}} & \frac{\Delta^{* 2}}{|\Delta|^{2}} L g_{\Delta_{1}, \Delta_{2}} \\
\frac{\Delta}{|\Delta|} L g_{\Delta_{2}, \alpha} & \frac{\Delta^{2}}{|\Delta|^{2}} L g_{\Delta_{1}, \Delta_{2}} & L g_{\Delta_{2}, \Delta_{2}}
\end{array}\right)
\end{aligned}
$$


where

$$
\begin{aligned}
L g_{\alpha, \alpha} & =\frac{-2 \widetilde{\omega}^{2}+\left(\epsilon_{1}+\epsilon_{2}-\epsilon_{3}-\epsilon_{4}\right) \widetilde{\omega}+2|\Delta|^{2}-\epsilon_{1} \epsilon_{2}-\epsilon_{3} \epsilon_{4}+\delta \omega\left(\epsilon_{2}-\epsilon_{3}-2 \widetilde{\omega}\right)}{\left(|\Delta|^{2}+\left(\epsilon_{2}-\widetilde{\omega}\right)\left(\epsilon_{3}+\widetilde{\omega}\right)\right)\left(|\Delta|^{2}-\left(\delta \omega-\epsilon_{1}+\widetilde{\omega}\right)\left(\delta \omega+\epsilon_{4}+\widetilde{\omega}\right)\right)} \\
L g_{\Delta_{1}, \Delta_{1}} & =\frac{\left(\epsilon_{2}-\omega\right)\left(\delta \omega+\epsilon_{4}+\widetilde{\omega}\right)}{\left(|\Delta|^{2}+\left(\epsilon_{2}-\widetilde{\omega}\right)\left(\epsilon_{3}+\widetilde{\omega}\right)\right)\left(|\Delta|^{2}-\left(\delta \omega-\epsilon_{1}+\widetilde{\omega}\right)\left(\delta \omega+\epsilon_{4}+\widetilde{\omega}\right)\right)} \\
L g_{\Delta_{2}, \Delta_{2}} & =\frac{-\left(\delta \omega-\epsilon_{1}+\omega\right)\left(\epsilon_{3}+\widetilde{\omega}\right)}{\left(|\Delta|^{2}+\left(\epsilon_{2}-\widetilde{\omega}\right)\left(\epsilon_{3}+\widetilde{\omega}\right)\right)\left(|\Delta|^{2}-\left(\delta \omega-\epsilon_{1}+\widetilde{\omega}\right)\left(\delta \omega+\epsilon_{4}+\widetilde{\omega}\right)\right)} \\
L g_{\Delta_{1}, \alpha} & =\frac{|\Delta|\left(\delta \omega+\epsilon_{2}+\epsilon_{4}\right)}{\left(|\Delta|^{2}+\left(\epsilon_{2}-\widetilde{\omega}\right)\left(\epsilon_{3}+\widetilde{\omega}\right)\right)\left(|\Delta|^{2}-\left(\delta \omega-\epsilon_{1}+\widetilde{\omega}\right)\left(\delta \omega+\epsilon_{4}+\widetilde{\omega}\right)\right)} \\
L g_{\Delta_{2}, \alpha} & =\frac{|\Delta|\left(-\delta \omega+\epsilon_{1}+\epsilon_{3}\right)}{\left(|\Delta|^{2}+\left(\epsilon_{2}-\widetilde{\omega}\right)\left(\epsilon_{3}+\widetilde{\omega}\right)\right)\left(|\Delta|^{2}-\left(\delta \omega-\epsilon_{1}+\widetilde{\omega}\right)\left(\delta \omega+\epsilon_{4}+\widetilde{\omega}\right)\right)} \\
L g_{\Delta_{1}, \Delta_{2}} & =\frac{-|\Delta|^{2}}{\left(|\Delta|^{2}+\left(\epsilon_{2}-\widetilde{\omega}\right)\left(\epsilon_{3}+\widetilde{\omega}\right)\right)\left(|\Delta|^{2}-\left(\delta \omega-\epsilon_{1}+\widetilde{\omega}\right)\left(\delta \omega+\epsilon_{4}+\widetilde{\omega}\right)\right)}
\end{aligned}
$$

One finds roots of the denominator and cast it in the form of product

$$
\begin{aligned}
& \left(\widetilde{\omega}-\sqrt{|\Delta|^{2}+\left(\frac{\epsilon_{2}+\epsilon_{3}}{2}\right)^{2}}+\frac{\epsilon_{3}-\epsilon_{2}}{2}-i o\right)\left(\widetilde{\omega}+\sqrt{|\Delta|^{2}+\left(\frac{\epsilon_{2}+\epsilon_{3}}{2}\right)^{2}}+\frac{\epsilon_{3}-\epsilon_{2}}{2}+i o\right) \\
& \left(\widetilde{\omega}-\sqrt{|\Delta|^{2}+\left(\frac{\epsilon_{1}+\epsilon_{4}}{2}\right)^{2}}+\delta \omega+\frac{\epsilon_{4}-\epsilon_{1}}{2}-i o\right)\left(\widetilde{\omega}+\sqrt{|\Delta|^{2}+\left(\frac{\epsilon_{1}+\epsilon_{4}}{2}\right)^{2}}+\delta \omega+\frac{\epsilon_{4}-\epsilon_{1}}{2}+i o\right),
\end{aligned}
$$

allowing for the correct rule of the poles circumvention, which implies that the virtual transition occur from the occupied states of the pairs below the gap to the empty states of the pairs above the gap.

Putting apart the terms of Eq.(29) in such a way that the frequency dependent factors in the denominator are grouped in pairs $\left(\widetilde{\omega}-\sqrt{|\Delta|^{2}+\left(\frac{\epsilon_{2}+\epsilon_{3}}{2}\right)^{2}}+\frac{\epsilon_{3}-\epsilon_{2}}{2}-i o\right) \times\left(\widetilde{\omega}+\sqrt{|\Delta|^{2}+\left(\frac{\epsilon_{1}+\epsilon_{4}}{2}\right)^{2}}+\delta \omega+\frac{\epsilon_{4}-\epsilon_{1}}{2}+i o\right)$, and $\left(\widetilde{\omega}+\sqrt{|\Delta|^{2}+\left(\frac{\epsilon_{2}+\epsilon_{3}}{2}\right)^{2}}+\frac{\epsilon_{3}-\epsilon_{2}}{2}+i o\right) \times\left(\widetilde{\omega}-\sqrt{|\Delta|^{2}+\left(\frac{\epsilon_{1}+\epsilon_{4}}{2}\right)^{2}}+\delta \omega+\frac{\epsilon_{4}-\epsilon_{1}}{2}-i o\right)$, and integrating over $d \widetilde{\omega}$, yields the matrix

$$
\begin{aligned}
\widehat{\widetilde{M}} & =\int d \widetilde{\omega} \frac{\partial^{2}}{\partial \ldots \partial \ldots} L g \\
& \equiv\left(\begin{array}{ccc}
\widetilde{M}_{\alpha, \alpha} & \frac{\Delta}{|\Delta|^{M}} \widetilde{M}_{\Delta_{1}, \alpha} & \frac{\Delta^{*}}{|\Delta|} \widetilde{M}_{\Delta_{2}, \alpha} \\
\frac{\Delta^{*}}{|\Delta|} \widetilde{M}_{\Delta_{1}, \alpha} & \widetilde{M}_{\Delta_{1}, \Delta_{1}} & \frac{\Delta^{* 2}}{|\Delta|^{2}} \widetilde{M}_{\Delta_{1}, \Delta_{2}} \\
\frac{\Delta}{|\Delta|} \widetilde{M}_{\Delta_{2}, \alpha} & \frac{\Delta^{2}}{|\Delta|^{2}} \widetilde{M}_{\Delta_{1}, \Delta_{2}} & \widetilde{M}_{\Delta_{2}, \Delta_{2}}
\end{array}\right)
\end{aligned}
$$

with the matrix elements

$$
\begin{gathered}
\widetilde{M}_{\alpha, \alpha}=-\frac{2 i \pi\left(4|\Delta|^{2}-\left(\epsilon_{2}+\epsilon_{3}\right)\left(\epsilon_{1}+\epsilon_{4}\right)+\eta_{1} \eta_{2}\right)}{\eta_{1} \eta_{2}\left(\delta \epsilon-2 \delta \omega+\eta_{1}+\eta_{2}\right)}-\frac{2 i \pi\left(4|\Delta|^{2}-\left(\epsilon_{2}+\epsilon_{3}\right)\left(\epsilon_{1}+\epsilon_{4}\right)+\eta_{1} \eta_{2}\right)}{\eta_{1} \eta_{2}\left(-\delta \epsilon+2 \delta \omega+\eta_{1}+\eta_{2}\right)} \\
\widetilde{M}_{\Delta_{1}, \Delta_{1}}=-\frac{i \pi\left(-\epsilon_{2}-\epsilon_{3}+\eta_{1}\right)\left(-\epsilon_{1}-\epsilon_{4}+\eta_{2}\right)}{\eta_{1} \eta_{2}\left(-\delta \epsilon+2 \delta \omega+\eta_{1}+\eta_{2}\right)}-\frac{i \pi\left(\epsilon_{2}+\epsilon_{3}+\eta_{1}\right)\left(\epsilon_{1}+\epsilon_{4}+\eta_{2}\right)}{\eta_{1} \eta_{2}\left(\delta \epsilon-2 \delta \omega+\eta_{1}+\eta_{2}\right)} \\
\widetilde{M}_{\Delta_{2}, \Delta_{2}}=-\frac{i \pi\left(-\epsilon_{2}-\epsilon_{3}+\eta_{1}\right)\left(-\epsilon_{1}-\epsilon_{4}+\eta_{2}\right)}{\eta_{1} \eta_{2}\left(\delta \epsilon-2 \delta \omega+\eta_{1}+\eta_{2}\right)}-\frac{i \pi\left(\epsilon_{2}+\epsilon_{3}+\eta_{1}\right)\left(\epsilon_{1}+\epsilon_{4}+\eta_{2}\right)}{\eta_{1} \eta_{2}\left(2 \delta \omega-\delta \epsilon+\eta_{1}+\eta_{2}\right)} \\
\widetilde{M}_{\Delta_{1}, \alpha}=\frac{2 i \pi \Delta\left(-\epsilon_{s}+\eta_{1}+\eta_{2}\right)}{\eta_{1} \eta_{2}\left(-\delta \epsilon+2 \delta \omega+\eta_{1}+\eta_{2}\right)}-\frac{2 i \pi \Delta\left(\epsilon_{s}+\eta_{1}+\eta_{2}\right)}{\eta_{1} \eta_{2}\left(\delta \epsilon-2 \delta \omega+\eta_{1}+\eta_{2}\right)} \\
\widetilde{M}_{\Delta_{2}, \alpha}=\frac{2 i \pi|\Delta|\left(-\epsilon_{s}+\eta_{1}+\eta_{2}\right)}{\eta_{1} \eta_{2}\left(\delta \epsilon-2 \delta \omega+\eta_{1}+\eta_{2}\right)}-\frac{2 i \pi \mid\left(\epsilon_{s}+\eta_{1}+\eta_{2}\right)}{\eta_{1} \eta_{2}\left(-\delta \epsilon+2 \delta \omega+\eta_{1}+\eta_{2}\right)} \\
\widetilde{M}_{\Delta_{1}, \Delta_{2}}=\frac{4 i \pi|\Delta|^{2}+\eta_{1}}{\eta_{1} \eta_{2}\left(\delta \epsilon-2 \delta \omega+\eta_{1}+\eta_{2}\right)}+\frac{4 i \pi|\Delta|^{2}}{\eta_{1} \eta_{2}\left(-\delta \epsilon+2 \delta \omega+\eta_{1}+\eta_{2}\right)}
\end{gathered}
$$

where the combinations $\eta_{2}=\sqrt{4|\Delta|^{2}+\left(\epsilon_{1}+\epsilon_{4}\right)^{2}}$ and $\eta_{1}=\sqrt{4|\Delta|^{2}+\left(\epsilon_{2}+\epsilon_{3}\right)^{2}}$ can be interpreted as energies of the initial and the virtual final states of the Cooper pair, respectively. The notations $\epsilon_{s}=\epsilon_{1}+\epsilon_{2}+\epsilon_{3}+\epsilon_{4}$, and $\delta \epsilon=\epsilon_{1}-\epsilon_{2}+\epsilon_{3}-\epsilon_{4}$ are introduced for shortness. 
Tracing in Eq.13) means that the expressions Eq. (31) containing energies $\epsilon_{i}$ should be integrated over the momenta $p_{r}$ and $\widetilde{k}$ and summed over the angular momentum $\widetilde{L}$. Performing this integration for the electron energy

$$
\epsilon_{f}(\widetilde{L}, \widetilde{k})=\frac{p_{r}^{2}}{2}+\frac{\widetilde{k}^{2}}{2}+\frac{(\widetilde{L}-\bar{L})^{2}}{2 R^{2}}-\mu,
$$

one can take into account that $\delta \epsilon=2 \delta L(\Lambda-\bar{L}) / R^{2}$ and employ different integration variables: $\xi$, $\varsigma$, and $L$, such that $\epsilon_{2}+\epsilon_{3}=2|\Delta| \sinh \xi, \epsilon_{1}+\epsilon_{4}=2|\Delta| \sinh \varsigma$, and $\widetilde{L}=L+\Lambda-\delta L / 2+\frac{|\Delta| \delta L(\sinh \varsigma-\sinh \xi)}{\delta k^{2}+\delta L^{2} / R^{2}}$, with the Jacobian

$$
J=\frac{4 R^{2}|\Delta|^{2} \cosh \zeta \cosh \xi}{(2 \pi)^{3} \sqrt{\Gamma-4 L^{2}\left(\delta L^{2}+R^{2} \delta k^{2}\right)}}
$$

where

$$
\Gamma=R^{4} \delta k^{2}\left[4|\Delta|\left(\sinh \zeta+\sinh \xi-\frac{|\Delta|}{\frac{\delta L^{2}}{R^{2}}+\delta k^{2}}(\sinh \xi-\sinh \zeta)^{2}\right)+\left(8 \mu-\delta k^{2}-\frac{\delta L^{2}+4(\bar{L}-\Lambda)^{2}}{R^{2}}\right)\right] .
$$

It includes the phase volume factor $(2 \pi)^{3}$ and an additional factor 2 allowing for the other brunch corresponding to the negative momenta $p_{r}$.

The variable $L$ enters only the Jacobian Eq.(33), and therefore the latter can be integrated over this variable within the domain where the square root is positive, thus yielding

$$
J_{L} \equiv \int \frac{d L}{R} \frac{4 R^{2}|\Delta|^{2} \cosh \zeta \cosh \xi}{(2 \pi)^{2} \sqrt{\Gamma-4 L^{2}\left(\delta L^{2}+R^{2} \delta k^{2}\right)}}=\int \frac{d X}{\sqrt{1-X^{2}}} \frac{2|\Delta|^{2} \cosh \zeta \cosh \xi}{(2 \pi)^{3} \sqrt{\left(\delta L^{2} / R^{2}+\delta k^{2}\right)}}=\frac{|\Delta|^{2} \cosh \zeta \cosh \xi}{(2 \pi)^{2} \sqrt{\frac{\delta L^{2}}{R^{2}}+\delta k^{2}}} .
$$

The contribution differs from zero only if $\Gamma>0$, which determines the integration domain $D[J]$ over the variables $\zeta$ and $\xi$ in Eq.(17). One thus arrives at

$$
\sinh \zeta+\sinh \xi-\frac{|\Delta|}{\frac{\delta L^{2}}{R^{2}}+\delta k^{2}}(\sinh \xi-\sinh \zeta)^{2}+\frac{2 \mu}{|\Delta|}>0,
$$

where the small term $-\delta k^{2}-\frac{\delta L^{2}+4(\bar{L}-\Lambda)^{2}}{R^{2}}$ is ignored as compared to $\mu$.

For the variables $\zeta=A+B / 2$ and $\xi=A-B / 2$, one can explicitly find the borders of the integration domain $D[J]$. In fact Eq.(35) in these variables reads

$$
2 \cosh \frac{B}{2} \sinh A-\frac{4|\Delta|}{\frac{\delta L^{2}}{R^{2}}+\delta k^{2}}\left(1+\sinh ^{2} A\right) \sinh ^{2} \frac{B}{2}+\frac{2 \mu}{|\Delta|}>0
$$

and determines borders for the variable $A$ :

$$
\frac{\cosh \frac{B}{2}-\sqrt{\cosh ^{2} \frac{B}{2}+16 \kappa^{2} \sinh ^{2} \frac{B}{2}\left(\frac{\mu}{|\Delta| 2 \kappa}-\sinh ^{2} \frac{B}{2}\right)}}{4 \kappa \sinh ^{2} \frac{B}{2}}<\sinh A<\frac{\cosh \frac{B}{2}+\sqrt{\cosh ^{2} \frac{B}{2}+16 \kappa^{2} \sinh ^{2} \frac{B}{2}\left(\frac{\mu}{|\Delta| 2 \kappa}-\sinh ^{2} \frac{B}{2}\right)}}{4 \kappa \sinh ^{2} \frac{B}{2}},
$$

and this condition implies real borders, that is

$$
\cosh ^{2} \frac{B}{2}+16 \kappa^{2} \sinh ^{2} \frac{B}{2}\left(\frac{\mu}{|\Delta| 2 \kappa}-\sinh ^{2} \frac{B}{2}\right)>0,
$$

where $\kappa=\frac{2|\Delta|}{\frac{\delta L^{2}}{R^{2}}+\delta k^{2}}$. Since $\cosh ^{2} \frac{B}{2}=1+\sinh ^{2} \frac{B}{2}$, one finds

$$
\frac{8 \kappa \frac{\mu}{|\Delta|}+1-\sqrt{64 \kappa^{2}+\left(8 \kappa \frac{\mu}{|\Delta|}+1\right)^{2}}}{32 \kappa^{2}}<\sinh ^{2} \frac{B}{2}<\frac{8 \kappa \frac{\mu}{|\Delta|}+1+\sqrt{64 \kappa^{2}+\left(8 \kappa \frac{\mu}{|\Delta|}+1\right)^{2}}}{32 \kappa^{2}} .
$$

The left part is negative and hence this inequality always holds, while the right part yields the integration domain over $B$. 
The matrix elements Eq.(31) now read

$$
\begin{gathered}
\widetilde{M}_{\alpha, \alpha}=-\widetilde{J}\left(\frac{2 i \pi(\cosh (\zeta-\xi)+1)}{-\Omega+\cosh \zeta+\cosh \xi}+\frac{2 i \pi(\cosh (\zeta-\xi)+1)}{\Omega+\cosh \zeta+\cosh \xi}\right) \\
\widetilde{M}_{\Delta_{1}, \Delta_{1}}=\widetilde{M}_{\Delta_{2}, \Delta_{2}}=-\widetilde{J}\left(\frac{i \pi e^{-\zeta-\xi}}{\Omega+\cosh \zeta+\cosh \xi}+\frac{i \pi e^{+\zeta \xi}}{-\Omega+\cosh \zeta+\cosh \xi}\right) \\
\widetilde{M}_{\Delta_{1}, \alpha}=-\widetilde{M}_{\Delta_{2}, \alpha}=\widetilde{J}\left(\frac{i \pi\left(e^{-\zeta}+e^{-\xi}\right)}{\Omega+\cosh \zeta+\cosh \xi}-\frac{i \pi\left(e^{\zeta}+e^{\xi}\right)}{-\Omega+\cosh \zeta+\cosh \xi}\right) \\
\widetilde{M}_{\Delta_{1}, \Delta_{2}}=\widetilde{J}\left(\frac{i \pi}{-\Omega+\cosh \zeta+\cosh \xi}+\frac{i \pi}{\Omega+\cosh \zeta+\cosh \xi}\right)
\end{gathered}
$$

where $\Omega=\frac{\delta \epsilon-2 \Delta \omega}{2|\Delta|}=\frac{\delta L(\Lambda-\bar{L}) / R^{2}-\Delta \omega}{\Delta}$ stands for the scaled and shifted perturbation frequency. One recognizes the structure of the integrals Eq.(17). The factor in front of the matrix elements

$$
\widetilde{J}=\frac{J_{L}}{2|\Delta| \cosh \zeta \cosh \xi}=\frac{|\Delta|}{8 \pi^{2} \sqrt{\frac{\delta L^{2}}{R^{2}}+\delta k^{2}}}
$$

originates from the Jacobian Eq.(34) and incorporates the factor $(2|\Delta|)^{-1}$, which makes the frequency $\Omega$ dimensionless. The phase of the order parameter does not enter in the final result and can be set to zero.

Now calculate the integrals

$$
\begin{aligned}
& \int_{D[J]} d \xi d \varsigma \frac{(\cosh (\zeta-\xi)+1)}{\Omega+\cosh (\zeta)+\cosh (\xi)}=\int_{-b}^{b} d B \int_{a_{-}}^{a_{+}} d A \frac{\cosh B+1}{\Omega+2 \cosh A \cosh \frac{B}{2}} \\
& \int_{D[J]} d \xi d \varsigma \frac{-\left(e^{-\zeta-\xi}+1\right)}{\Omega+\cosh (\zeta)+\cosh (\xi)}=-\int_{-b}^{b} d B \int_{a_{-}}^{a_{+}} d A \frac{e^{-2 A}+1}{\Omega+2 \cosh A \cosh \frac{B}{2}} \\
& \int_{D[J]} d \xi d \varsigma \frac{\left(e^{-\zeta}+e^{-\xi}\right)}{\Omega+\cosh \zeta+\cosh \xi}=2 \int_{-b}^{b} d B \int_{a_{-}}^{a_{+}} d A \frac{e^{A} \cosh \frac{B}{2}}{\Omega+2 \cosh A \cosh \frac{B}{2}} \\
& a_{ \pm}=\operatorname{arcsinh} \frac{\cosh \frac{B}{2} \pm \sqrt{\cosh ^{2} \frac{B}{2}+16 \kappa^{2} \sinh ^{2} \frac{B}{2}\left(\frac{\mu}{|\Delta| 2 \kappa}-\sinh ^{2} \frac{B}{2}\right)}}{4 \kappa \sinh ^{2} \frac{B}{2}} \\
& b=2 \operatorname{arcsinh} \sqrt{\frac{\sqrt{64 \kappa^{2}+\left(8 \kappa \frac{\mu}{\Delta}+1\right)^{2}}+8 \kappa \frac{\mu}{|\Delta|}+1}{32 \kappa^{2}}}
\end{aligned}
$$

where $D[J]$ is the domain restricted by the condition Eq.(35) which is explicitly given for the variables $A$ and $B$, as it is shown above. One finds

$$
\begin{gathered}
\left.4 \int_{-b}^{b} d B \frac{\arctan \left(\frac{\left(2 \cosh \frac{B}{2}-\Omega\right) \tanh \frac{A}{2}}{\sqrt{4 \cosh ^{2} \frac{B}{2}-\Omega^{2}}}\right) \cosh ^{2} \frac{B}{2}}{\sqrt{4 \cosh ^{2} \frac{B}{2}-\Omega^{2}}}\right|_{A=a_{-}} ^{A=a_{+}}, \\
\left.\int_{-b}^{b} d B\left(\frac{\Omega^{2} \arctan \frac{\Omega+2 e^{-A} \cosh \frac{B}{2}}{\sqrt{4 \cosh ^{2} \frac{B}{2}-\Omega^{2}}}}{\sqrt{4 \cosh ^{2}\left(\frac{B}{2}\right)-\Omega^{2} \cosh ^{2} \frac{B}{2}}}+\frac{e^{-A}}{\cosh \frac{B}{2}}-\Omega \frac{\log \left(\Omega+2 \cosh A \cosh \frac{B}{2}\right)-A}{2 \cosh ^{2} \frac{B}{2}}\right)\right|_{A=a_{-}} ^{A=a_{+}}, \\
\left.\int_{-b}^{b} d B\left(A+\log \left(\Omega+2 \cosh A \cosh \frac{B}{2}\right)-\frac{2 \Omega \arctan \frac{\Omega+2 e^{A} \cosh \frac{B}{2}}{\sqrt{4 \cosh ^{2} \frac{B}{2}}}}{\sqrt{4 \cosh ^{2} \frac{B}{2}-\Omega^{2}}}\right)\right|_{A=a_{-}} ^{A=a_{+}},
\end{gathered}
$$

for the first, the second and the third integrals Eq.(38), respectively. Integration over $d B$ has to be done numerically. Results of the numerical calculations are shown in the following figures. 


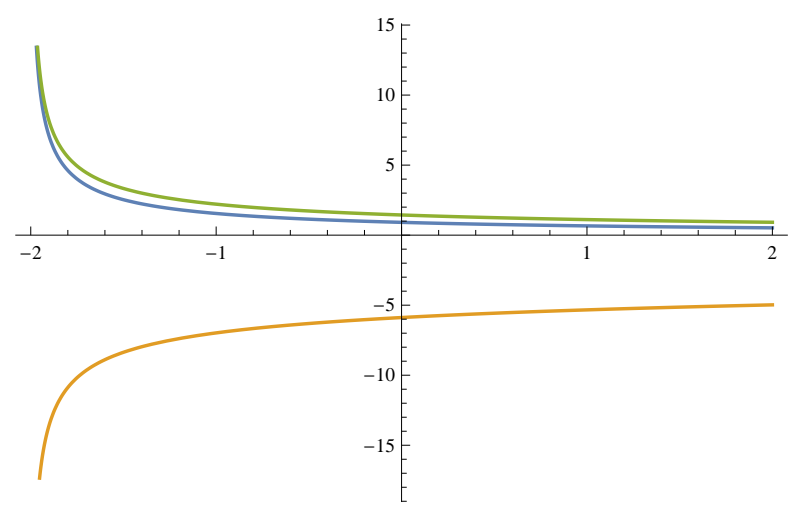

Integrals that after symmetrization or antisymmetrization give Eq. (38) as functions of $\Omega$ in the interval from $\Omega=-2$ till $\Omega=2$. The parameters are: $\frac{\mu}{|\Delta|} \simeq 580$ and $\kappa \simeq 22275$. The integral $\mathcal{I}_{1}(\Omega)$ corresponds to the blue curve, $\mathcal{I}_{2}(\Omega)$ is negative (corresponds to the orange color), and $\mathcal{I}_{3}(\Omega)$ corresponds to the green curve. The dependencies on $\frac{\mu}{|\Delta|}$ and on $\kappa$ are very weak, having logarithmic character, shown for $\mathcal{I}_{2}\left(\Omega=0, \frac{\mu}{|\Delta|}, \kappa\right)$ in Fig IV

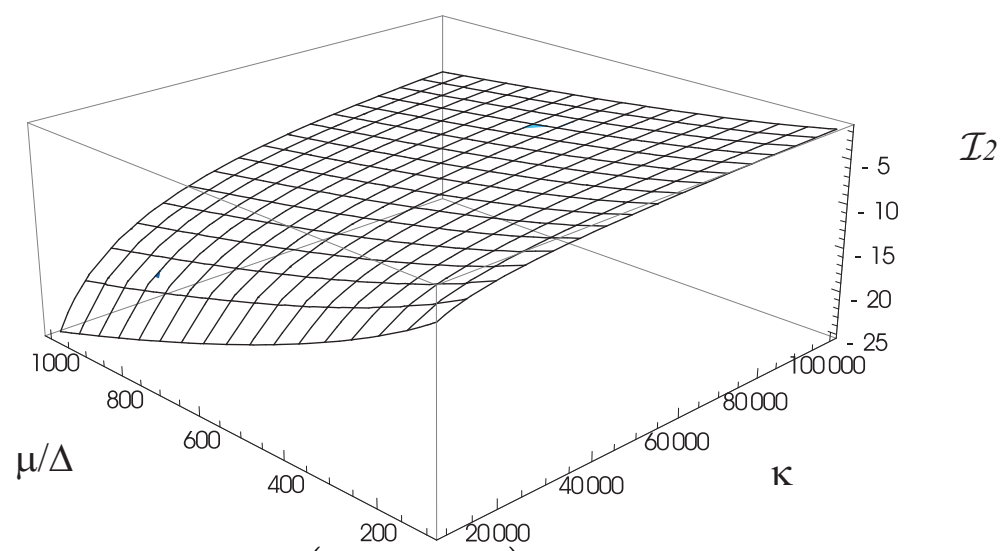

Dependence of $\mathcal{I}_{2}\left(\Omega=0, \frac{\mu}{|\Delta|}, \kappa\right)$ on the parametrs $\frac{\mu}{|\Delta|}$ and $\kappa$.

Combining Eqs.(15]30[36]17]37]28) with Eq.(27), one arrives at

$$
\begin{aligned}
& \frac{|\Delta| i \pi}{8 \pi \sqrt{\frac{\delta L^{2}}{R^{2}}+\delta k^{2}}} \times \\
& \left(\begin{array}{lll}
\alpha^{*} & \Delta_{1} & \Delta_{2}^{*}
\end{array}\right)\left(\begin{array}{ccc}
-2 \mathcal{I}_{1}(\Omega) \int\left|\vec{u}_{k}^{*} \vec{u}_{k^{\prime}}\right|^{2} n_{e} d V & \frac{\Delta}{|\Delta|} \mathcal{I}_{3}(\Omega) \int \frac{\left(\vec{u}_{k}^{*} \vec{u}_{k^{\prime}}\right) n_{e} d V}{\sqrt{2 \pi d R l}} & -\frac{\Delta^{*}}{|\Delta|} \mathcal{I}_{3}(\Omega) \int \frac{\left(\vec{u}_{k}^{*} \vec{u}_{k^{\prime}}\right) n_{e} d V}{\sqrt{2 \pi d R l}} \\
\frac{\Delta^{*}}{|\Delta|} \mathcal{I}_{3}(\Omega) \int \frac{\left(\vec{u}_{k^{\prime}}^{*} \vec{u}_{k}\right) n_{e} d V}{\sqrt{2 \pi d R l}} & \left(\mathcal{I}_{2}(\Omega)+\mathcal{I}_{4}(\Omega) \int \frac{n_{e} d V}{2 \pi d R l}\right. & \mathcal{I}_{4}(\Omega) \int \frac{n_{e} d V}{2 \pi d R l} \frac{\Delta^{* 2}}{|\Delta|^{2}} \\
-\frac{\Delta}{|\Delta|} \mathcal{I}_{3}(\Omega) \int \frac{\left(\vec{u}_{k^{\prime}}^{*} \vec{u}_{k}\right) n_{e} d V}{\sqrt{2 \pi d R l}} & \frac{\Delta^{2}}{|\Delta|^{2}} \mathcal{I}_{4}(\Omega) \int \frac{n_{e} d V}{2 \pi d R l} & \left(\mathcal{I}_{2}(\Omega)+\mathcal{I}_{4}(\Omega)\right) \int \frac{n_{e} d V}{2 \pi d R l}
\end{array}\right)\left(\begin{array}{c}
\alpha \\
\Delta_{1}^{*} \\
\Delta_{2}
\end{array}\right) .
\end{aligned}
$$

After performing the integration over the volume with the allowance for Eq.(9) one finds

$$
\frac{i|\Delta| n_{e}}{8 \pi \sqrt{\frac{\delta L^{2}}{R^{2}}+\delta k^{2}}}\left(\begin{array}{lll}
\alpha^{*} & \Delta_{1} & \Delta_{2}^{*}
\end{array}\right)\left(\begin{array}{ccc}
-2 \mathcal{I}_{1}(\Omega) O_{p} & \frac{\Delta}{|\Delta|} \mathcal{I}_{3}(\Omega) O_{p o} & -\frac{\Delta^{*}}{|\Delta|} \mathcal{I}_{3}(\Omega) O_{p o} \\
\frac{\Delta^{*}}{|\Delta|} \mathcal{I}_{3}(\Omega) O_{p o}^{*} & \mathcal{I}_{2}(\Omega)+\mathcal{I}_{4}(\Omega) & \mathcal{I}_{4}(\Omega) \frac{\Delta^{* 2}}{|\Delta|^{2}} \\
-\frac{\Delta}{|\Delta|} \mathcal{I}_{3}(\Omega) O_{p o}^{*} & \frac{\Delta^{2}}{|\Delta|^{2}} \mathcal{I}_{4}(\Omega) & \mathcal{I}_{2}(\Omega)+\mathcal{I}_{4}(\Omega)
\end{array}\right)\left(\begin{array}{c}
\alpha \\
\Delta_{1}^{*} \\
\Delta_{2}
\end{array}\right),
$$

where

$$
\begin{aligned}
O_{p} & =\int\left|\vec{u}_{k}^{*} \vec{u}_{k^{\prime}}\right|^{2} d V=\frac{(\pi v / c)^{2}}{2 \omega_{k^{\prime}} \omega_{k}} \frac{\pi R d\left(\vec{u}_{k}^{*}(R) \cdot \vec{u}_{k^{\prime}}(R)\right)^{2}}{l} \\
O_{p o} & =\int \frac{\left(\vec{u}_{k}^{*} \vec{u}_{k^{\prime}}\right) d V}{\sqrt{2 \pi d R l}}=\frac{\pi v / c}{\sqrt{2 \omega_{k} \omega_{k^{\prime}}}} \frac{\sqrt{\pi R d}\left(\vec{u}_{k}^{*}(R) \cdot \vec{u}_{k^{\prime}}(R)\right)}{\sqrt{l}} \\
O_{o} & =1
\end{aligned}
$$


are the overlap integrals of the mode functions in the domain occupied by the superconductor.

The phases of the unperturbed order parameter and the phase difference of the field modes can be included to the phases of $\alpha, \Delta_{1}$ and $\Delta_{2}$, which yields

$$
\frac{i|\Delta| n_{e}}{8 \pi \sqrt{\frac{\delta L^{2}}{R^{2}}+\delta k^{2}}}\left(\begin{array}{ccc}
\alpha^{*} & \Delta_{1} & \Delta_{2}^{*}
\end{array}\right)\left(\begin{array}{ccc}
-2 \mathcal{I}_{1}(\Omega) O_{p} & \mathcal{I}_{3}(\Omega) O_{p o} & -\mathcal{I}_{3}(\Omega) O_{p o} \\
\mathcal{I}_{3}(\Omega) O_{p o}^{*} & \mathcal{I}_{2}(\Omega)+\mathcal{I}_{4}(\Omega) & \mathcal{I}_{4}(\Omega) \\
-\mathcal{I}_{3}(\Omega) O_{p o} & \mathcal{I}_{4}(\Omega) & \mathcal{I}_{2}(\Omega)+\mathcal{I}_{4}(\Omega)
\end{array}\right)\left(\begin{array}{c}
\alpha \\
\Delta_{1}^{*} \\
\Delta_{2}
\end{array}\right) .
$$

The action now reads

$$
i\left(\begin{array}{ccc}
\alpha^{*} & \Delta_{1} & \Delta_{2}^{*}
\end{array}\right)\left(\begin{array}{ccc}
-2 \nu \mathcal{I}_{1}(\Omega) O_{p} & \nu \mathcal{I}_{3}(\Omega) O_{p o} & -\nu \mathcal{I}_{3}(\Omega) O_{p o} \\
\nu \mathcal{I}_{3}(\Omega) O_{p o} & \nu \mathcal{I}_{2}(\Omega)+\nu \mathcal{I}_{4}(\Omega)+\frac{1}{2 g} & \nu \mathcal{I}_{4}(\Omega) \\
-\nu \mathcal{I}_{3}(\Omega) O_{p o} & \nu \mathcal{I}_{4}(\Omega) & \nu \mathcal{I}_{2}(\Omega)+\nu \mathcal{I}_{4}(\Omega)+\frac{1}{2 g}
\end{array}\right)\left(\begin{array}{c}
\alpha \\
\Delta_{1}^{*} \\
\Delta_{2}
\end{array}\right)
$$

where

$$
\nu=\frac{|\Delta| n_{e}}{8 \pi \sqrt{\frac{\delta L^{2}}{R^{2}}+\delta k^{2}}}
$$

and the Gaussian integration over $d \Delta_{1} d \Delta_{2}$ gives

$$
Z\left(\alpha^{*}, \alpha\right)=\text { const } \exp \left[\frac{-|\Delta| n_{e} \alpha^{*} \alpha}{4 \pi \sqrt{\frac{\delta L^{2}}{R^{2}}+\delta k^{2}}}\left(O_{p} \mathcal{I}_{1}(\Omega)+\frac{\mathcal{I}_{3}^{2}(\Omega) O_{p o}^{2}\left(1-\delta_{0}^{\delta L}\right)}{\frac{4 \pi \sqrt{\delta L^{2} / R^{2}+\delta k^{2}}}{g|\Delta| n_{e}}+\mathcal{I}_{2}(\Omega)}\right)\right]
$$

and after taking the derivative Eq.(51) finally yields

$$
\chi_{k, k^{\prime}, \bar{k}^{\prime}, \bar{k}}=\frac{-|\Delta| n_{e}}{4 \pi \sqrt{\frac{\delta L^{2}}{R^{2}}+\delta k^{2}}}\left(O_{p} \mathcal{I}_{1}(\Omega)+\frac{\mathcal{I}_{3}^{2}(\Omega) O_{p o}^{2}\left(1-\delta_{0}^{\delta L}\right)}{\frac{4 \pi \sqrt{\delta L^{2} / R^{2}+\delta k^{2}}}{g|\Delta| n_{e}}+\mathcal{I}_{2}(\Omega)}\right),
$$

or explicitly

$$
\chi_{k, k^{\prime}, \bar{k}^{\prime}, \bar{k}}=\frac{-|\Delta| n_{e} \pi^{2}}{8 \sqrt{\frac{\delta L^{2}}{R^{2}}+\delta k^{2}}} \frac{R d\left(\vec{u}_{k}^{*}(R) \cdot \vec{u}_{k^{\prime}}(R)\right)^{2}}{(c / v)^{2} \omega_{k^{\prime}} \omega_{k} l}\left(\mathcal{I}_{1}(\Omega)+\frac{\mathcal{I}_{3}^{2}(\Omega)\left(1-\delta_{0}^{\delta L}\right)}{\frac{4 \pi \sqrt{\delta L^{2} / R^{2}+\delta k^{2}}}{g|\Delta| n_{e}}+\mathcal{I}_{2}(\Omega)}\right)
$$

Presence of the Kronekker delta $\delta_{0}^{\delta L}$ is due to the fact that for the case $\delta L=0$, one finds $\Delta_{1}=\Delta_{2}^{*}$, which results in the fact that the collective mode becomes forbidden for the Raman transition and cannot be excited. Finally, one finds the nonlinear susceptibility

$$
\chi_{k, k^{\prime}, \bar{k}^{\prime}, \bar{k}}=\frac{-|\Delta| n_{e} d\left(\vec{u}_{k}^{*}(R) \cdot \vec{u}_{k^{\prime}}(R)\right)^{2}}{32 R \sqrt{\frac{\delta L^{2}}{R^{2}}+\delta k^{2}}(c / v)^{2} \omega_{k^{\prime}} \omega_{k} l} h(\Omega)
$$

which couples photons with the wave-vectors $k, k^{\prime}, \bar{k}^{\prime}, \bar{k}$ satisfying the condition $k-k^{\prime}=\bar{k}-\bar{k}^{\prime}=\delta k, L-L^{\prime}=\bar{L}-\bar{L}^{\prime}=$ $\delta L$. The frequency profile reads

$$
h(\Omega)=\mathcal{I}_{1}(\Omega)+\frac{\left(1-\delta_{\delta L}^{0}\right) \mathcal{I}_{3}^{2}(\Omega)}{\frac{4 \pi \sqrt{\delta L^{2} / R^{2}+\delta k^{2}}}{g|\Delta| n_{e}}+\mathcal{I}_{2}(\Omega)} .
$$

In Eq.(40), in contrast to Eq.(39), the factor $\left(\vec{u}_{k}^{*}(R) \cdot \vec{u}_{k^{\prime}}(R)\right)^{2}$ has been replaced by $\frac{\left(\vec{u}_{k}^{*}(R) \cdot \vec{u}_{k^{\prime}}(R)\right)^{2}}{\left(2 \pi R_{i n n}\right)^{2}}$ to allow for the normalization of the radial mode functions of the photons. With this expression one can substitute the scalar product $\left(\vec{u}_{k}^{*}(R) \cdot \vec{u}_{k^{\prime}}(R)\right)=-0.4$ that has been found earlier for the radius scaled to unity.

The collective mode exists when the equation

$$
\frac{4 \pi \sqrt{\delta L^{2} / R^{2}+\delta k^{2}}}{g|\Delta| n_{e}}+\mathcal{I}_{2}(\Omega)=0
$$

has solutions for $\Omega^{2}<4$. This happens if $\frac{4 \pi \sqrt{\delta L^{2} / R^{2}+\delta k^{2}}}{g|\Delta| n_{e}}>-\mathcal{I}_{2}(\Omega=0)$. However, due to the logarithmic character of the divergency of $\mathcal{I}_{2}(\Omega)$ at $\Omega^{2} \rightarrow 4$, the collective mode turns out to be exponentially close to the gap boarders, when the left side of this equality becomes much smaller as compared to the right side. 


\section{APPENDIX}

The collective mode exists when the equation

$$
\frac{4 \pi \sqrt{\delta L^{2} / R^{2}+\delta k^{2}}}{g|\Delta| n_{e}}+\mathcal{I}_{2}(\Omega)=0
$$

has solutions for $\Omega^{2}<4$. This happens if Eq.(20) $\frac{4 \pi \sqrt{\delta L^{2} / R^{2}+\delta k^{2}}}{g|\Delta| n_{e}}+\mathcal{I}_{2}(\Omega=0)>0$ holds. This condition depends on the typical "size " $1 / \sqrt{\delta L^{2} / R^{2}+\delta k^{2}}$ of the waveguide and on the properties of the superconductors. By employing the relations

$$
\begin{aligned}
g[\text { a.u. }] & \simeq \frac{20.045}{n_{e}^{1 / 3}[\text { a.u. }] \log \left(\frac{861330 . n_{e}^{2 / 3}[a . u .]}{T_{c}\left[K^{\circ}\right]}\right)} \\
\Delta[\text { a.u. }] & \simeq 5.555 \times 10^{-6} T_{c}\left[K^{\circ}\right] \\
\mu[\text { a.u. }] & \simeq \frac{1}{2} 3^{2 / 3} \pi^{4 / 3} n_{e}^{2 / 3}[\text { a.u. }]
\end{aligned}
$$

one finds the domains where the collective modes exist and are reasonably far from the gap borders. This domain depends both on the superconducting material properties and on its typical size $1 / \sqrt{\delta k^{2}+\delta L^{2} / R^{2}}$ given by the radius $R$ of the tube and the mode wavenumber difference $\delta k$. In the following figure

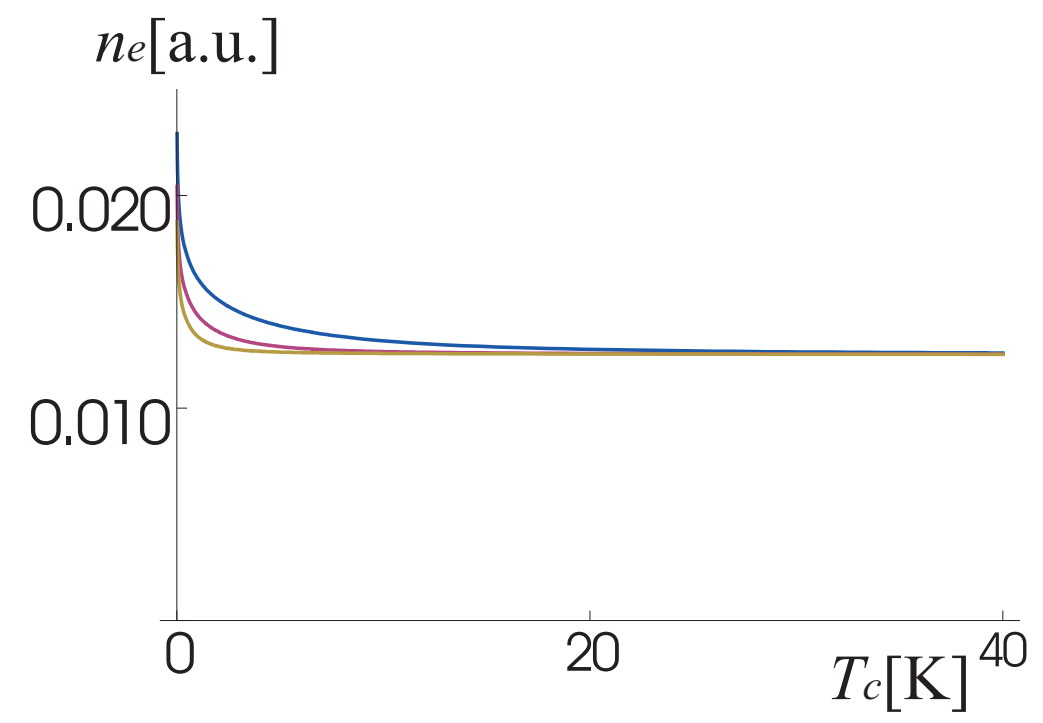

Domains of the critical temperatures (absis axis) and the electron densities (ordinate axis, in atomic units $0.01[a . u.] \simeq 0.7 \times 10^{23} \mathrm{~cm}^{-3}$ ) where the collective mode is possible are above the curves. The curves correspond to typical sizes $1 / \sqrt{\delta k^{2}+\delta L^{2} / R^{2}}$ of the waveguides with $\delta k=0.225 \mu^{-1}$ and $R$ equals to $1 \mu$ (top), $3 \mu$ (middle), and $10 \mu$ (bottom).

one sees this dependence for tube waveguides of radii 1,3 , and $10 \operatorname{microns}(\mu)$ and for the mode wavenumber difference $\sim 0.2 \mu^{-1}$.

\section{APPENDIX}

Since the nonlinear susceptibility for $\delta L=0$ practically does not depend on the wavevector within the mode, the amplitudes for $m=1, m^{\prime}=-1$ and for $m=-1, m^{\prime}=1$ in the antisymmetric combination cancel nonlinearity each of the other in such a way that this combination does not experience action of the nonlinearity. For the symmetric combination, on the contrary, the nonlinear coupling given by $\chi_{\delta L=0} l$ acts. The coupling is local in $z$ and does not depend on the "quantization length" $l$. By the analogy, the amplitudes for $m=-1, m^{\prime}=-1$ and for $m=1, m^{\prime}=1$ experience action of the local nonlinear couplings $\chi_{\delta L=-2} l$ and $\chi_{\delta L=2} l$, respectively. 
As long as the dependence $k(\omega)$ is restricted to the linear terms of the Taylor expansions accounting just for the group velocities, the Schrödinger equation for the amplitudes in the coordinate representation (given by the corresponding Fourier transformation of that in the momentum representation) belongs to the class of the first order differential equations

$$
\left(-i \frac{\partial}{\partial t}-i v \frac{\partial}{\partial z}-i v^{\prime} \frac{\partial}{\partial z^{\prime}}+A\left(z-z^{\prime}\right)\right) \Phi\left(t, z, z^{\prime}\right)=0
$$

in 3 dimensional space $\left(t, z, z^{\prime}\right)$ and therefore can be solved by the method of characteristics yielding the general solution of the form

$$
\Phi\left(t, z, z^{\prime}\right)=\Phi\left(z-v t, z^{\prime}-v^{\prime} t\right) e^{-\frac{i}{2} \int^{z-z^{\prime}} A(x) d x},
$$

where $\bar{\Phi}(x, y)$ is an arbitrary function of two variables has to be found from the initial conditions. For two independent bell-shaped accident wave packets $\bar{\Phi}(x, y)=\phi(x) \bar{\phi}(y)$ with no initial overlap, that is $\int \phi(z-v t) \bar{\phi}\left(z-v^{\prime} t\right) d z=0$ for $t<t_{\text {in }}$, the asymptotic solution for $t \rightarrow \infty$ reads

$$
\Phi\left(t, z, z^{\prime}\right)=\phi(z-v t) \bar{\phi}\left(z^{\prime}-v^{\prime} t\right) e^{\frac{-i}{2} \int_{-\infty}^{\infty} A(t \delta v) d t} .
$$

For the interaction independent on the wavevector, the function $A\left(z-z^{\prime}\right)$ is local, that is proportional to the Dirac delta function $A \delta_{z-z^{\prime}}$, and the asymptotic form attains after a finite interval of time $t>t_{\text {fin }}$ when the faster wavepacket completely overtakes the slower one, such that

$$
\int \phi\left(z-v t_{f i n}\right) \bar{\phi}\left(z-v^{\prime} t_{f i n}\right) d z=0 .
$$

[1] A. Nakanishi H. Katayama-Yoshida, Solid State Communications, 152, 24-27 (2012)

[2] A.Kumatani,T.Ohsawa, R. Shimizu, Y. Takagi,S. Shiaki, T. Hitosugi, Appl. Phys.Lett,101,123103 (2012).

[3] R.H. Dicke, Phys. Rev. 93, 99-110 (1954)

[4] A.A. Abrikosov, L.P. Gorkov, I.E. Dzyaloshinski, Methods of Quantum Field Theory in Statistical Physics, Richard A. Silverman, ISBN-10:0-486-63228-8, pp. 315320, (1963)

[5] I. O. Kulik O. Entin-Wohlman, R. Orbach, Journal of Low Temperature Physics 43, Issue 5-6, pp 591-620, (1981)

[6] M.V. Klein, S.B. Dierker, Phys.Rev.B 29, 4976-4991 (1984)
[7] For details one can consult, for instance, the review by I. N. Toptygin, Physics-Uspekhi, 60, 935-947 (2017)

[8] J.H. Eberly, J. Javanainen, K. Rzazevski, Physics Reports, 204,331-383 (1991)

[9] For the details see the book by H.Kleinert, Collective Classical and Quantum Fields World Scientific 2018, ISBN: 978-981-3223-93-6, Sec. 3.1

[10] Eq. (7) is the Fourier representation of Eqs.(3.45-47) of [9] obtrained from the action $\int d t \operatorname{Tr} \vec{\psi}+\left(i \partial_{t}-\widehat{H}\right) \vec{\psi}$ with the help of anticommutation and integration in parts.

[11] A.J. Leggett, Progress of Theoretical Physics, 36, 901930 (1966) 